\title{
Synaptic Integration of Functionally Diverse Pallidal Information in the Entopeduncular Nucleus and Subthalamic Nucleus in the Rat
}

\author{
Mark D. Bevan, Nick P. Clarke, and J. Paul Bolam \\ Medical Research Council Anatomical Neuropharmacology Unit and University Department of Pharmacology, \\ Oxford OX1 3TH, United Kingdom
}

To determine the principles of synaptic innervation of neurons in the entopeduncular nucleus and subthalamic nucleus by neurons of functionally distinct regions of the pallidal complex, double anterograde labeling was carried out at both light and electron microscopic levels in the rat. Deposits of the anterograde tracers Phaseolus vulgaris-leucoagglutinin and biotinylated dextran amine were placed in different functional domains of the pallidal complex in the same animals. The tracer deposits in the ventral pallidum and the globus pallidus gave rise to GABA-immunopositive projections to the entopeduncular nucleus, the subthalamic nucleus, and the more medial lateral hypothalamus that were largely segregated but overlapped at the interface between the two fields of projection. In these regions the proximal parts of individual neurons in the entopeduncular nucleus, lateral hypothalamus, and subthalamic nucleus received synaptic input from terminals derived from both the ventral pallidum and the globus pallidus. Furthermore, the analysis of the afferent synaptic input to the dendrites of neurons in the subthalamic nucleus that cross functional boundaries of the nucleus defined by the pallidal inputs, revealed that terminals with the morphological and neurochemical characteristics of those derived from the pallidal complex make synaptic contact with all parts of the dendritic tree, including distal regions.

It is concluded that functionally diverse information carried by the descending projections of the pallidal complex is synaptically integrated by neurons of the entopeduncular nucleus, lateral hypothalamus, and subthalamic nucleus by two mechanisms. First, neurons located at the interface between functionally distinct, but topographically adjacent, projections could integrate diverse information by means of the synaptic convergence at the level of the cell body and proximal dendrites. Second, because the distal dendrites of neurons in the subthalamic nucleus receive input from the pallidum, those that extend across two distinct domains of pallidal input could also provide the morphological basis of integration.

Key words: ventral pallidum; globus pallidus; subthalamic nucleus; entopeduncular nucleus; basal ganglia; synaptic convergence
The basal ganglia are a group of subcortical nuclei involved in various processes, including motor, associative, cognitive, and mnemonic functions. A major role of the basal ganglia is to integrate sensorimotor, associative, and limbic information to produce context-dependent behaviors (Nauta and Domesick, 1984; Graybiel, 1995), a function that is evident from behavioral and physiological analyses (Wurtz and Hikosaka, 1986; Schultz et al., 1993; Graybiel et al., 1994; Aosaki et al., 1995). The basal ganglia receive topographical projections from all functional territories of the cortex that are directed to the neostriatum and its ventral homolog the nucleus accumbens (Kunzle, 1975; Goldman and Nauta, 1977; Van Hoesen et al., 1981; Nauta and Domesick, 1984; Selemon and Goldman-Rakic, 1985; Alexander et al., 1986, 1990; McGeorge and Faull, 1989; Flaherty and Graybiel, 1991; Hoover and Strick, 1993; Groenewegen and Berendse, 1994; Joel

\footnotetext{
Received Aug. 13, 1996; accepted Oct. 2, 1996.

This work was funded by the Wellcome Trust and the Medical Research Council, United Kingdom. N.P.C. is in receipt of a Medical Research Council Studentship; M.D.B. is in receipt of a Wellcome Trust Advanced Training Fellowship (046613/2/ 96/2). We thank Caroline Francis, Paul Jays, Frank Kennedy, and Liz Norman for technical assistance, Peter Somogyi for the GABA antiserum, Claudio Cuello for the antibodies to substance P, Véronique Bernard and Jason Hanley for comments on this manuscript, and David Smith for helpful discussions throughout the period of the work.

Correspondence should be addressed to J. P. Bolam, Medical Research Council Anatomical Neuropharmacology Unit, University Department of Pharmacology, Mansfield Road, Oxford OX1 3TH, UK.

Copyright (C) 1996 Society for Neuroscience $\quad 0270-6474 / 96 / 170308-17 \$ 05.00 / 0$
}

and Weiner, 1994). Although the results of anatomical analyses suggest that the segregation of functional information imparted by the topographical cortical input is maintained at each level of a series of segregated, parallel basal ganglia-thalamocortical loops (Alexander et al., 1986, 1990; Hoover and Strick, 1993; Groenewegen and Berendse, 1994; Joel and Weiner, 1994; Lynch et al., 1994), it is clear that systems must exist within these loops where integration of the diverse information occurs at the synaptic level. Several sites or systems have been proposed to subserve this function, including the local circuit neurons of the neostriatum and nucleus accumbens (Gerfen, 1984; Chesselet and Graybiel, 1986; Kubota and Kawaguchi, 1993; Bolam and Bennett, 1995), the ascending projections of midbrain dopamine neurons (Somogyi et al., 1981; Nauta and Domesick, 1984; Gerfen et al., 1987; Jimenez-Castellanos and Graybiel, 1987; Smith and Bolam, 1990), the pattern of striatal innervation of neurons of the globus pallidus and substantia nigra (Francois et al., 1984; Percheron et al., 1984, 1987; Yelnik et al., 1984), the innervation of the pedunculopontine region by the internal segment of the globus pallidus (Smith and Shink, 1995), and "open" cortico-basal ganglia-thalamocortical loops (Joel and Weiner, 1994). We have recently demonstrated an additional system that is part of the indirect pathway of information flow through the basal ganglia, which may underlie a powerful mechanism for the integration of diverse information in the basal ganglia (Bevan et al., 1995b, 1996). By double 
anterograde tracing we demonstrated that neurons of functionally diverse regions of the pallidal complex, i.e., globus pallidus (motor and associative) and the ventral pallidum (limbic), made convergent synaptic contacts both with neurons of the substantia nigra pars reticulata (basal ganglia output neurons) and with the dopamine neurons of the ventral midbrain, in addition to providing segregated inputs to the substantia nigra. The innervation by both types of pallidal neurons took the form that is typical of pallidal innervation described previously (Smith and Bolam, 1989, 1991; Bolam et al., 1993), i.e., both sets of terminals formed basket-like, multiple, GABA-positive symmetrical synaptic contacts with the cell body and proximal dendrites of their targets.

Neurons of the pallidal complex, as part of the indirect pathway of information flow through the basal ganglia (Albin et al., 1989; Alexander and Crutcher, 1990; DeLong, 1990), in addition to projecting to the substantia nigra also project to the other output nucleus of the basal ganglia, i.e., the entopeduncular nucleus (Kincaid et al., 1991; Bolam and Smith, 1992) and the subthalamic nucleus (DeLong, 1990). The question we address in this report is whether the same rules of synaptic innervation of neurons in the substantia nigra by neurons of the pallidal complex also apply to the innervation of neurons in the entopeduncular nucleus and subthalamic nucleus. In particular, we have looked for evidence of convergence of synaptic input from functionally distinct regions of the pallidal complex onto the same individual neurons in each of these two target nuclei.

Some of the data reported in this manuscript have been published previously in abstract form (Bevan and Bolam, 1995a; Bevan et al., 1995b; Clarke et al., 1996a).

\section{MATERIALS AND METHODS}

Preparation of tissue. All surgical procedures were carried out on male Sprague Dawley rats (250-350 gm) under deep anesthesia, which was induced and maintained by injection of pentobarbitone (Sagatal, 60 $\mathrm{mg} / \mathrm{kg}$, i.p.). Injections of neuronal tracers were placed into different regions of the pallidal complex under stereotaxic guidance, using coordinates derived from the atlas of Paxinos and Watson (1986). The rats were maintained on a $12 \mathrm{hr}$ light/dark cycle, with free access to food and water. Environmental conditions for housing of the rats and all procedures that were performed on them were in accordance with the Animals (Scientific Procedures) Act 1986 and with the policy on the use of animals in neuroscience research issued by the Society for Neuroscience.

Double anterograde tracing combined with pre- and post-embedding immunocytochemistry at the light and electron microscopic levels was performed in eight rats as described previously (Smith and Bolam, 1991), except that biotinylated dextran amine (BDA) was used as one of the tracers (Veenman et al., 1992). While under deep anesthesia, the rats received iontophoretic injections (5-9 $\mu \mathrm{A}, 7 \mathrm{sec}$ on/7 sec off, $20 \mathrm{~min}$ ) of Phaseolus vulgaris-leucoagglutinin [PHA-L; $2.5 \%$ in $0.01 \mathrm{M}$ phosphate buffer (PB), pH 8.0; Vector Laboratories, Peterborough, UK] (Gerfen and Sawchenko, 1984) in the ventral pallidum and biotinylated dextran amine (10\% in $0.9 \%$ sodium chloride; Molecular Probes, Eugene, OR) in the globus pallidus. In one case the injections were reversed.

After a survival period of 1 week, the animals were reanesthetized, perfused with $100 \mathrm{ml}$ PBS (0.01 M phosphate, $\mathrm{pH} 7.4)$ for 1-2 min, and then perfused with $300 \mathrm{ml}$ of $0.1-0.5 \%$ glutaraldehyde and $2-3 \%$ paraformaldehyde in PB (0.1 M, pH 7.4) for 20 min, followed by $100-200 \mathrm{ml}$ of $2-3 \%$ paraformaldehyde for $10 \mathrm{~min}$. After fixation the brain was removed from the cranium, divided into 5-mm-thick coronal slices, and stored in PBS at $4^{\circ} \mathrm{C}$ before further processing. From each slice, $60 \mu \mathrm{m}$ coronal sections through the injection sites, the striatal complex, the subthalamic nucleus, and the entopeduncular nucleus were taken by a vibrating microtome and collected in PBS. Sections for electron microscopy were freeze-thawed as described previously (von Krosigk and Smith, 1991 ) and then washed several times in PBS before further processing. Sections for light microscopy alone had $0.3 \%$ Triton X-100 included in the diluent for the antibodies and peroxidase complex.
Visualization of neuronal tracers. A series of sections were processed for the light microscopic visualization of both tracers. The BDA was visualized by incubation of the sections in an avidin-biotin-peroxidase complex (1:100 dilution; Vector Laboratories) in PBS containing 1\% bovine serum albumin at room temperature for $4 \mathrm{hr}$ or overnight at $4^{\circ} \mathrm{C}$. Peroxidase linked to BDA by the avidin-biotin bridge was revealed by placing the sections in Tris buffer $(0.05 \mathrm{M}, \mathrm{pH} 7.6)$ containing $0.025 \% 3,3^{\prime}$ diaminobenzidine tetrahydrochloride (DAB) and $0.006 \%$ hydrogen peroxide for 10-15 min. The reaction was terminated by rinsing the sections several times in Tris buffer.

The PHA-L was then visualized by incubating the sections in rabbit anti-PHA-L antibody (1:1000 dilution; Dako, High Wycombe, UK) overnight at room temperature or for $48 \mathrm{hr}$ at $4^{\circ} \mathrm{C}$. After many washes in PBS, the sections were incubated in a solution of goat anti-rabbit IgG (1:100; Dako) at room temperature for $2 \mathrm{hr}$ followed by a $2 \mathrm{hr}$ incubation in rabbit peroxidase anti-peroxidase complex (1:100; Dako). Bound peroxidase was revealed by incubation in hydrogen peroxide $(0.00075 \%)$, using DAB $(0.015 \%)$ in the presence of nickel ammonium sulfate $(0.5 \%)$ as the chromogen. The antisera were diluted in PBS containing $1 \%$ bovine serum albumin and $2 \%$ normal goat serum.

Alternate sections through the injection sites were processed to reveal substance P [1:50 rat anti-substance P (Cuello et al., 1979); 1:100 goat anti-rat, Dako; 1:100 rat PAP, Incstar) immunoreactivity, using DAB as the chromogen for the peroxidase reaction (see above). Substance P is a marker of the ventral pallidum and was used to determine the precise location of the injection sites (Haber and Nauta, 1983; Groenewegen et al., 1993).

The majority of the sections was processed for correlated light and electron microscopic analysis. All antibody incubations and peroxidase reactions were carried out as described above, except that Triton X-100 was not included in the diluent and benzidine dihydrochloride (BDHC) was used as the chromogen to visualize PHA-L-labeled structures (Smith and Bolam, 1991, 1992; Bevan et al., 1994a). The strict safety precautions described by Yung et al. (1996) were used when the BDHC as well as the DAB were handled.

Processing of sections for correlated light and electron microscopy. After visualization of the tracers, the sections containing anterogradely transported tracers were placed flat at the bottom of a Petri dish and post-fixed in $1 \%$ osmium tetroxide (Oxchem, $\mathrm{UK}$ ) (in $0.01 \mathrm{M} \mathrm{PB}$ at $\mathrm{pH} 6.8$ ) for 20-30 min. The sections were then washed in $\mathrm{PB}$ and rapidly dehydrated through a graded series of dilutions of ethanol. To enhance the contrast of the tissue in the electron microscope, the sections were stained with $1 \%$ uranyl acetate (Taab) at the $70 \%$ ethanol phase. The sections were washed twice for $10 \mathrm{~min}$ in propylene oxide and placed in resin (Durcupan; Fluka, Buchs, Switzerland) overnight. Finally, the sections were embedded in resin on microscope slides, placed in an oven, and cured for $48 \mathrm{hr}$ at $60^{\circ} \mathrm{C}$.

Analysis of material. The sections that included the injection sites were examined in the light microscope, and the location and extent of the tracer injections in the pallidal complex were assessed and compared with the distribution of substance $\mathrm{P}$ immunoreactivity in the adjacent sections. The anterograde labeling arising from the tracer deposits was plotted with the aid of a drawing tube. Particular note was made of neurons that were apposed by anterogradely labeled boutons derived from both tracer deposits. Sections prepared for correlated light and electron microscopy were examined in the light microscope, and individual neurons that were apposed by both sets of anterogradely labeled terminals in the entopeduncular nucleus, lateral hypothalamus, or subthalamic nucleus, or regions that contained a large number of both types of terminals were photographed, cut out from the slides, and glued to blank resin blocks. Serial ultrathin sections were cut on a Reichert-Jung Ultracut-E ultramicrotome and collected on Pioloformcoated copper or gold single-slot grids. The ultrathin sections were contrasted with lead citrate for 1-2 min and examined in a Phillips CM10 or 410 electron microscope.

Synaptology of neurons of the subthalamic nucleus. To characterize the afferent synaptology of neurons of the subthalamic nucleus, seven rats received iontophoretic deposits (5-10 $\mu \mathrm{A}, 7 \mathrm{sec}$ on/7 sec off, $15 \mathrm{~min})$ of the tracer neurobiotin (5\% in $0.9 \% \mathrm{NaCl}$; Vector Laboratories) (Kita and Armstrong, 1991; Lapper and Bolam, 1991) in the substantia nigra. After a survival time of 1 day, the animals were perfused and sectioned as described above. Sections of the subthalamic nucleus were incubated to reveal the injected and transported tracer, and the tissue was prepared for correlated light and electron microscopy essentially as described for BDA. Individual neurons in the subthalamic nucleus that were labeled 

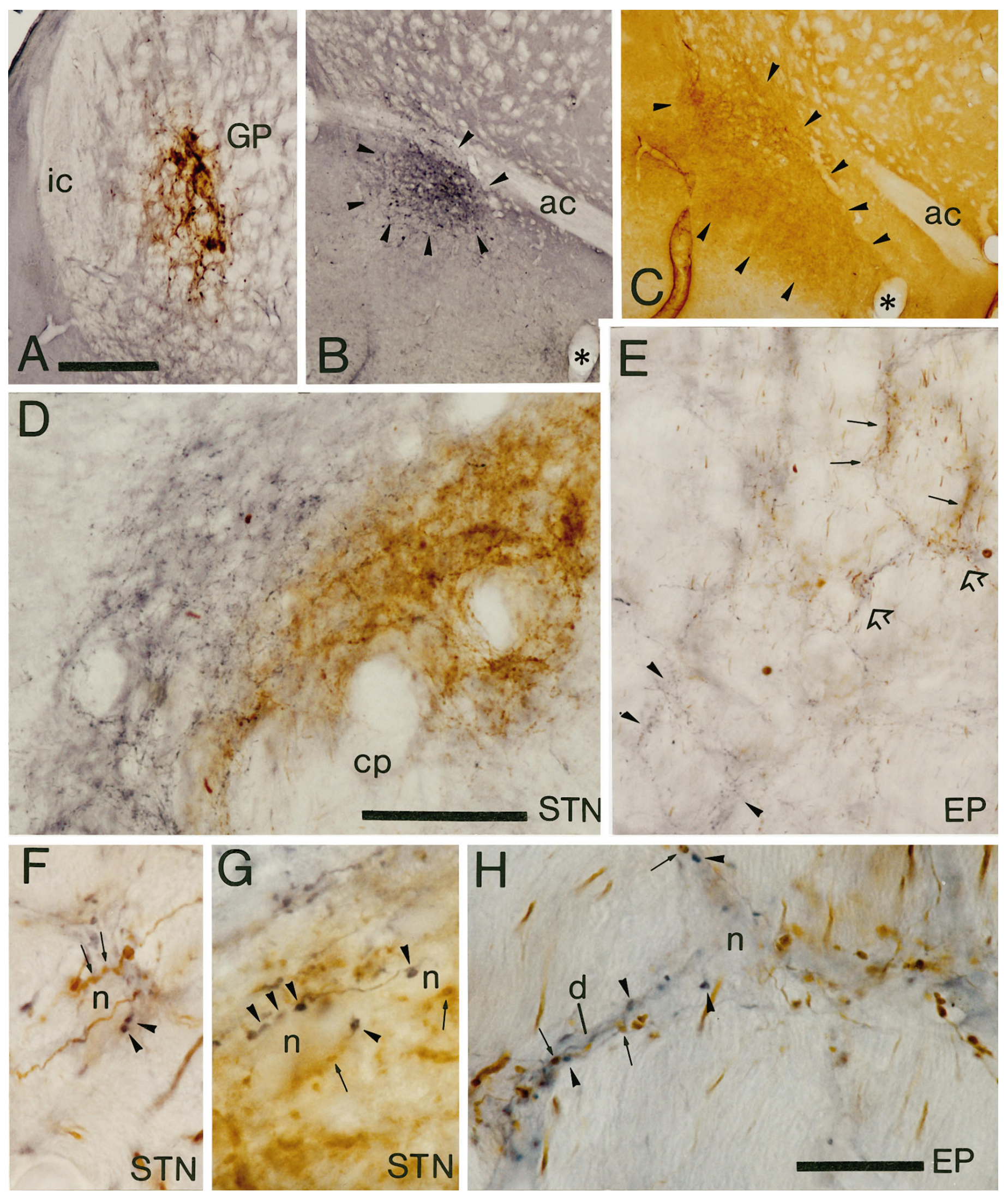

Figure 1. Light micrographs of anterograde labeling from the ventral pallidum and globus pallidus $(G P)$ in the entopeduncular nucleus $(E P)$ and subthalamic nucleus $(S T N)$. $A-C$, Sites of deposit of $\mathrm{BDA}$ in the globus pallidus $(A)$ were revealed by using DAB as the chromogen, and PHA-L in the ventral pallidum $(B)$ were revealed by using nickel-DAB as the chromogen for the peroxidase reaction. The extent of the tracer deposit in the ventral pallidum (arrowheads) was assessed by staining of adjacent sections to reveal substance $\mathrm{P}$ immunoreactivity (arrowheads) $(C)$. $D$, Medium-power micrograph of anterograde labeling in the subthalamic nucleus. The fibers anterogradely labeled from the ventral pallidum (blue) (Figure legend continues) 
A

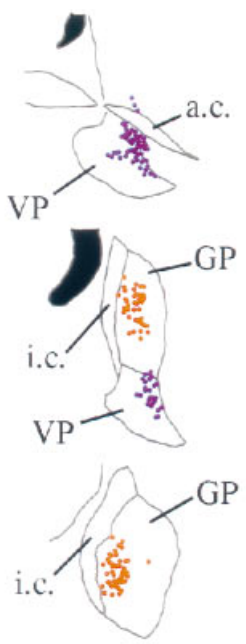

B
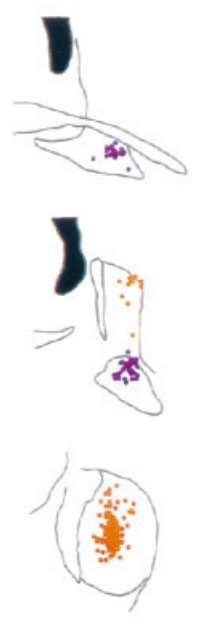

C

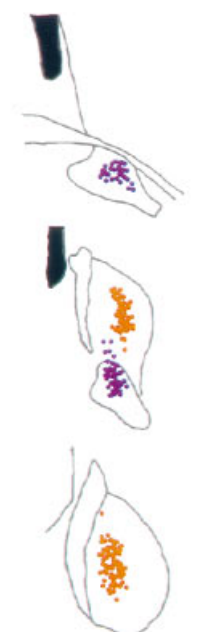

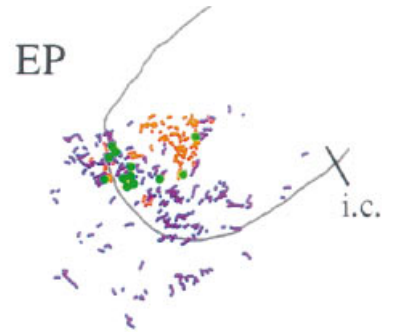
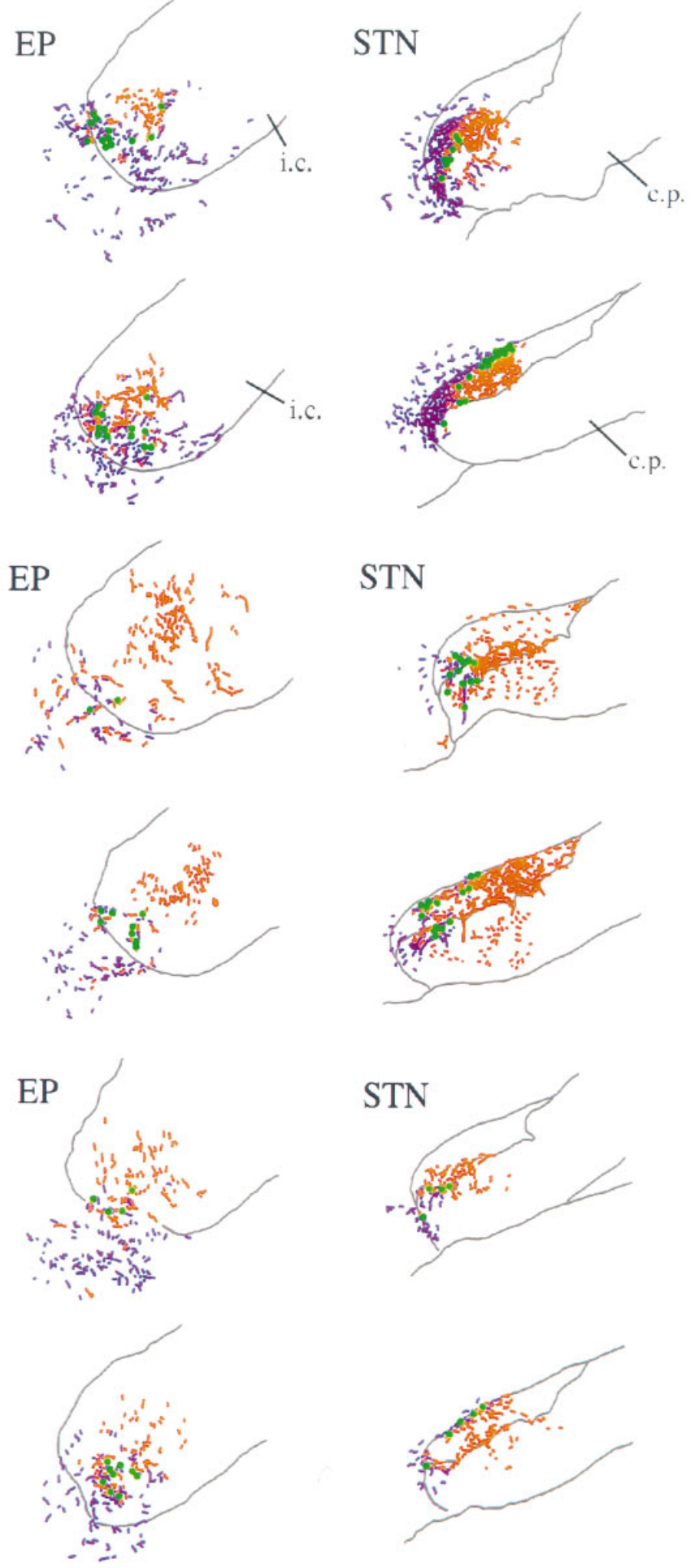

Figure 2. Schematic representations of the sites of deposit of PHA-L in the ventral pallidum $(V P)$ and BDA in the globus pallidus $(G P)$ and the site of anterograde transport in the entopeduncular nucleus $(E P)$ and subthalamic nucleus $(S T N)$ in three animals $(A-C)$. Dots at the injection sites represent individual neurons that have taken up the PHA-L (blue) or the BDA (red). The blue and red stippling of the two rostrocaudal levels of the $E P$ and $S T N$ represent the anterogradely labeled fibers from the $V P$ and $G P$, respectively. Although the topography of the two projections is distinct, many neurons were identified (green dots) that were apposed by boutons derived from both the $V P$ and $G P$. $a c$, Anterior commissure; $c p$, cerebral peduncle; $i c$, internal capsule.

$\leftarrow$

occupy the medial and dorsal aspects of the STN, whereas those from the globus pallidus (brown) occupy the more lateral parts; however, the two sets of anterogradely labeled fibers are mixed at the interface between the projections. Note that the width of the fields of anterograde labeling is well within the dendritic diameter of subthalamic neurons. E, Medium-power micrograph of anterograde labeling in the entopeduncular nucleus. The fibers derived from the globus pallidus (brown; some indicated by arrows) and those derived from the ventral pallidum (blue; some indicated by arrowheads) are largely separate at this level, although there are areas of overlap of the two sets of fibers (some indicated by open arrows and shown at higher magnification in $H) . F, G$, High-power micrographs of unstained neuronal perikarya in the STN that are closely apposed by axonal swellings derived from both the ventral pallidum (blue; some indicated by arrowheads) and the globus pallidus (brown; some indicated by arrows). Individual perikarya are apposed by several boutons from each site. $H$, High-power micrograph of the region of the entopeduncular nucleus indicated by open arrows in $E$. An unstained neuronal perikaryon $(n)$ and dendrite $(d)$ are closely apposed by axonal swellings derived from both the ventral pallidum (blue; some indicated by arrowheads) and the globus pallidus (brown; some indicated by arrows). $a c$, Anterior commissure; $c p$, cerebral peduncle; $i c$, internal capsule. Scale bars: $A-C$ (shown in $A$ ), $500 \mu \mathrm{m} ; D, E$, (shown in $D), 100 \mu \mathrm{m} ; F-H$ (shown in $H$ ), $20 \mu \mathrm{m}$. 


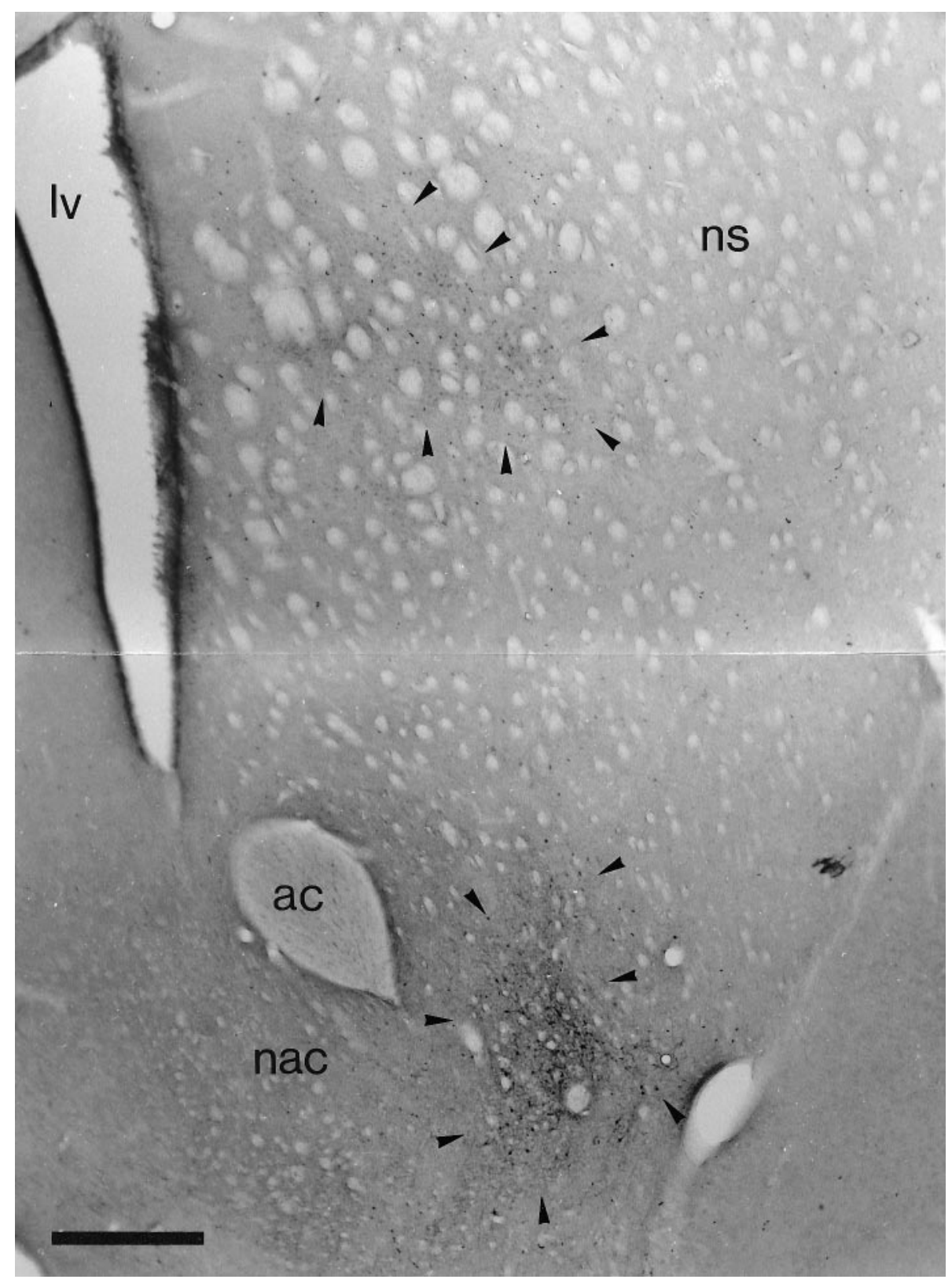

Figure 3. Retrograde labeling in the striatal complex after tracer deposits in the ventral pallidum and the globus pallidus. Low-power micrograph of the neostriatum and nucleus accumbens of the same animal as illustrated in Figure $2 C$. The section was incubated to reveal retrogradely transported PHA-L that was injected in the ventral pallidum and BDA that was injected in the globus pallidus. Although it is difficult to distinguish the labeling in this black and white micrograph, neurons retrogradely labeled from the globus pallidus (labeled with DAB; area indicated by arrowheads) are present only in the dorsal part of the neostriatum, whereas neurons retrogradely labeled from the ventral pallidum (labeled with $\mathrm{Ni}$ $\mathrm{DAB}$; area indicated by arrowheads) are present only in the most ventral aspects of the neostriatum and the nucleus accumbens. $a c$, Anterior commissure; $l v$, lateral ventricle; nac, nucleus accumbens; $n s$, neostriatum. Scale bar, $0.5 \mathrm{~mm}$.

extensively with the neurobiotin were identified, drawn, photographed, and re-embedded for electron microscopic analysis. Serial sections were collected and examined in the electron microscope. The position of each terminal forming synaptic contact with the subthalamic neurons was noted. The terminals were classified according to their morphology and the nature of the postsynaptic specialization. The presence of GABA in the synaptic boutons was then tested by post-embedding immunocytochemistry (see below). Cross-sectional areas of labeled terminals were determined from photographs, using a digitizing pad and MacStereology software.

Post-embedding immunocytochemistry for GABA. To test for the presence of fixed GABA in anterogradely labeled and nonlabeled terminals in the entopeduncular nucleus, lateral hypothalamus, and subthalamic nucleus, ultrathin sections were collected on gold grids and labeled by the post-embedding immunogold method. The method was a slight modification of that described by Phend and colleagues (1992). The grids were first washed in $0.05 \mathrm{M}$ Tris buffer, $\mathrm{pH} 7.6$, containing $0.9 \% \mathrm{NaCl}$ and $0.01 \%$ Triton X-100 (TBS-Triton) and then incubated overnight at room temperature on drops of a 1:5000-15,000 dilution of a rabbit anti-GABA antiserum (code 9; Hodgson et al., 1985; Somogyi and Hodgson, 1985; Somogyi et al., 1985) in TBS-Triton. After several washes in TBS-Triton and one wash in TBS at $\mathrm{pH} 8.2$, the grids were incubated for $1-1.5 \mathrm{hr}$ at room temperature in a 1:25 dilution of $15 \mathrm{~nm}$ gold-conjugated goat

Figure 4. Synaptic convergence of terminals derived from different functional domains of the pallidal complex in the entopeduncular nucleus. $A$, Electron micrograph of part of a proximal dendrite of a neuron in the entopeduncular nucleus $(E P n)$. The neuron is apposed by three anterogradely labeled boutons, each of which forms symmetrical synaptic contact with the neuron (arrows). Two of the boutons contain the BDHC peroxidase reaction product that was used to localize the PHA-L anterogradely transported from the ventral pallidum $\left(b_{V P}\right)$. The third bouton $\left(b_{G P}\right)$ contains the DAB reaction product that was used to localize the BDA anterogradely transported from the globus pallidus. Note that the BDHC reaction product that labels the terminals from the ventral pallidum has an irregular appearance and occupies only part of the labeled bouton, leaving many vesicles visible that do not have reaction product associated with them. In contrast, the DAB reaction product that labels the boutons from the globus pallidus is amorphous and occupies the whole of the labeled structure. B, A serial section of the upper of the two boutons derived from the ventral pallidum. This (Figure legend continues) 


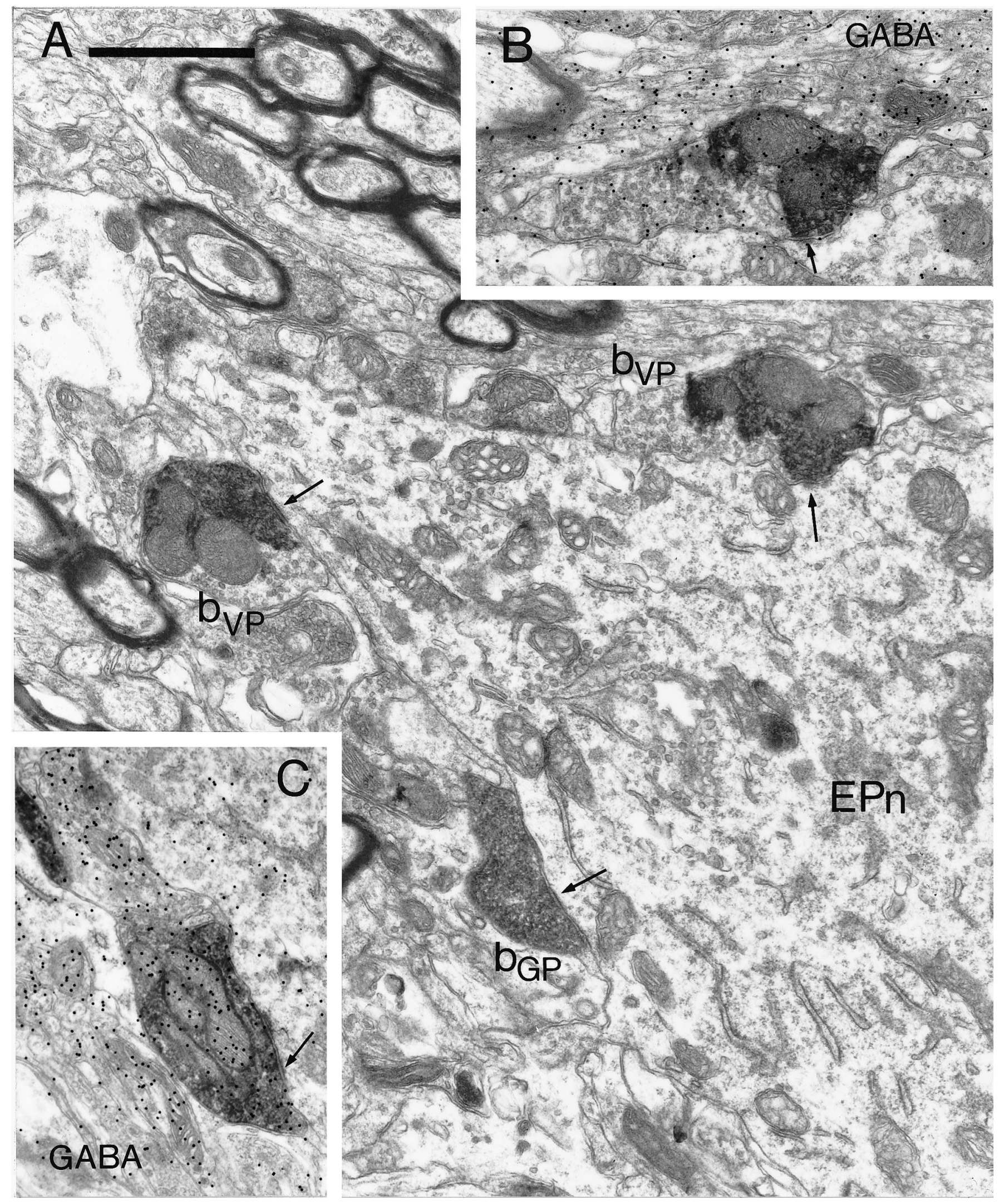

section was processed by the post-embedding immunogold method to reveal GABA immunoreactivity. The bouton has a high density of immunogold particles associated with it (index of GABA immunoreactivity $=3.79$ ). $C$, Serial section of the bouton derived from the globus pallidus labeled by the post-embedding immunogold method to reveal GABA immunoreactivity. The bouton has a high density of immunogold particles associated with it (index of GABA immunoreactivity $=7.51$ ). Scale bar (shown in $A$ ): $A-C, 1 \mu \mathrm{m}$. 
anti-rabbit IgG (BioCell, Cardiff, UK) in TBS at pH 8.2. The grids were washed in TBS at $\mathrm{pH} 8.2$ and then in water and stained with $1 \%$ aqueous uranyl acetate for $1-1.5 \mathrm{hr}$ and then with lead citrate. The sections were then examined in the electron microscope.

Immunoreactivity for GABA was detected by the presence of the electron-dense immunogold particles overlying labeled structures. To quantify the immunoreactivity, the density (particles $/ \mu \mathrm{m}^{2}$ ) of immunogold particles overlying individual structures was calculated. The density of labeling overlying terminals was corrected for nonspecific binding of the antibody to tissue-free resin by subtracting the density of gold particles overlying the lumen of capillaries in the same ultrathin section. The index of GABA immunoreactivity for each bouton was then calculated by normalizing the corrected density with respect to the labeling associated with GABA-immunonegative terminals forming asymmetrical synapses on the same ultrathin section.

\section{RESULTS \\ Light microscopic analysis}

Location of injection sites

The PHA-L was revealed as a blue reaction product produced by the nickel/DAB, and the $\mathrm{BDA}$ as a brown reaction product produced by the DAB. The sites of injection of PHA-L into the ventral pallidum or BDA into the globus pallidus (or the reverse in a single case) were characterized by densely labeled neurons surrounded by a halo of diffuse extracellular staining (Fig. 1A,B). A total of eight cases that had injections located discretely in both divisions of the pallidal complex were used in this analysis. Some of these animals have also been used in an analysis of the pallidal innervation of the substantia nigra (Bevan et al., 1996). In each case the deposits of tracers in the ventral pallidum were confined to the subcommissural region of the pallidal complex and included both the lateral and medial subdivisions (Figs. $1 B, 2$; also see illustrations in Bevan et al., 1996). The localization of the deposits within the ventral pallidum was confirmed by substance P immunostaining of sections adjacent to those incubated to reveal the tracer (Fig. 1C). Rarely, small numbers of labeled neurons were observed in the transitional zone where the boundary between the globus pallidus and ventral pallidum is difficult to define. The deposits of tracers in the globus pallidus were confined to that nucleus and did not encroach on the ventral pallidum or the medially placed internal capsule (Figs. $1 A, 2$ ). In two cases, small numbers of neurons were labeled in the neostriatum along the injection tract. In most of the cases the deposits were confined to the central region of the GP in its rostral two thirds.

The deposits of either BDA or PHA-L in ventral pallidum or globus pallidus gave rise to retrograde labeling of neurons in the striatal complex in addition to anterograde labeling (see below). The deposits of tracer in the ventral pallidum gave rise to retrogradely labeled neurons in the nucleus accumbens and most ventral parts of the neostriatum, whereas the deposits of tracer in the globus pallidus gave rise to retrograde labeling in the dorsal parts of the neostriatum (Fig. 3).

\section{Anterograde labeling}

Each of the deposits of tracer in the ventral pallidum or the globus pallidus gave rise to anterograde labeling in the neostriatum, nucleus accumbens, entopeduncular nucleus, lateral hypothalamus, subthalamic nucleus, substantia nigra/ventral tegmental area, and thalamus. The anterograde labeling in the ventral mesencephalon is the subject of separate communications (Bevan et al., 1996) and will thus not be dealt with further. The anterograde labeling followed a topographical ordering that was consistent with previous observations (Smith and Bolam, 1989, 1991; Groenewegen et al., 1993). Tracer deposits in the globus pallidus led to anterograde labeling that was largely confined to the more dorsal and lateral parts of the entopeduncular nucleus, whereas the tracer deposits in the ventral pallidum led to anterograde labeling in the more medial and ventral parts of the entopeduncular nucleus that extended into the lateral hypothalamus (Fig. 2). Similarly, in the subthalamic nucleus, anterograde labeling derived from the globus pallidus was located more laterally than were anterogradely labeled fibers derived from the ventral pallidum (Figs. 1E, 2). The tracer deposits in the ventral pallidum gave rise to labeling in the medial and dorsal subthalamic nucleus and also extended medially into the lateral hypothalamus (Figs. 1D, 2).

Despite the topographic separation of the majority of anterogradely labeled structures derived from the ventral pallidum and the globus pallidus, zones of overlap were observed in the entopeduncular nucleus/lateral hypothalamus and the medial and dorsal subthalamic nucleus (Figs. $1 D, E, 2$ ).

At high magnification the morphology and organization of the anterogradely labeled fibers and boutons were consistent with previous observations (Haber et al., 1985; Smith et al., 1990; Bolam and Smith, 1992; Groenewegen et al., 1993). Thus the labeled structures derived from either the globus pallidus or the ventral pallidum gave rise to a dense meshwork of fibers (Fig. $1 D, E$ ) that possessed large boutons (Fig. $1 F-H)$. The boutons often gave rise to a basket-like innervation of unstained neuronal perikarya (Fig. $1 F-H$ ). In regions of overlap between the two sets of anterogradely labeled structures, fibers and axonal swellings were closely intermixed. Boutons derived from both the ventral pallidum and the globus pallidus were often apposed to the same unstained neuron (Fig. $1 F-H)$.

\section{Electron microscopic analysis}

Because it is not possible to distinguish $\mathrm{DAB}$ and nickel/DAB reaction products at the electron microscopic level, the electron microscopic analysis was conducted on material in which the anterogradely transported PHA-L was revealed by using BDHC as the chromogen for the peroxidase reaction, and the BDA was revealed by using $\mathrm{DAB}$ as the chromogen. The two reaction products are readily distinguishable at the electron microscopic level (Levey et al., 1986; Smith and Bolam, 1991; Bevan et al., 1994a,b). The DAB reaction product presumably forms as a precipitate during the peroxidase reaction; it is thus floccular and adheres to the external surface of organelle membranes, including vesicles and mitochondria and the internal surface of the plasma membrane (Figs. $4 A, C, 5 B, 6 F, 7 B, 8 C$ ). It is distributed characteristically throughout the labeled structure. In lightly labeled structures, it appears as an electron-dense material lining membranes (Figs. $5 B, 7 B$ ), but in more heavily labeled structures it appears as an amorphous material that fills the whole of the structure, often obscuring the internal details (Figs. 4A, 6F, 8C). The BDHC reaction product, in contrast, appears to be crystalline, having an irregular, nonfloccular appearance (Figs. 4A, $B, 5 C$, $6 C-E, 7 C, D, 8 B)$. As such, the reaction product does not adhere to membranes and is commonly found in only part of the labeled structure, leaving regions free of reaction product (Figs. $4 A, B, 5 C$, $6 C-E, 8 B)$. The BDHC reaction product is often more electrondense than the DAB reaction product (Fig. 4).

\section{Terminals derived from the globus pallidus}

Consistent with previous observations (Smith et al., 1990; Bolam and Smith, 1992), the terminals derived from the globus pallidus in both the entopeduncular nucleus and the subthalamic nucleus 
Table 1. Postsynaptic targets of pallidal terminals

\begin{tabular}{|c|c|c|c|c|c|}
\hline \multirow[b]{2}{*}{ Source } & & & \multicolumn{3}{|c|}{$\begin{array}{l}\text { Postsynaptic target } \\
\text { (\% distribution) }\end{array}$} \\
\hline & \multicolumn{2}{|c|}{ Target region } & Perikarya & $\begin{array}{l}\text { Large } \\
\text { dendrites }\end{array}$ & $\begin{array}{l}\text { Small } \\
\text { dendrites }\end{array}$ \\
\hline Ventral pallidum & $\mathrm{EP} / \mathrm{LH}$ & (71) & 31 & 19.7 & 49.3 \\
\hline Globus pallidus $^{a}$ & EP & $(162)$ & 12.8 & 23.3 & 64 \\
\hline Ventral pallidum & STN/LH & (90) & 21 & 18.9 & 60 \\
\hline Globus pallidus $^{b}$ & STN & (187) & 31 & 39 & 30 \\
\hline Globus pallidus $^{c}$ & $\mathrm{SNr}$ & $(105)$ & 54 & 32 & 14 \\
\hline
\end{tabular}

Postsynaptic targets of terminals derived from the ventral pallidum in the entopeduncular/lateral hypothalamus (EP/LH) and the subthalamic nucleus/lateral hypothalamus $(\mathrm{STN} / \mathrm{LH})$ obtained in this study compared with data on terminals from the globus pallidus obtained in other studies. Large dendrites and small dendrites refer to those dendrites with a diameter greater or smaller than $1.5 \mu \mathrm{m}$ in diameter in the $\mathrm{EP} / \mathrm{LH}$ and substantia nigra reticulata $(\mathrm{SNr})$ and $1 \mu \mathrm{m}$ in the STN/LH. Figures represent the percentage of the number of terminals shown in brackets in contact with different elements.

Data obtained from ${ }^{a}$ Bolam and Smith, 1992; ${ }^{b}$ Smith et al., 1990; ${ }^{c}$ Smith and Bolam, 1991.

were large and usually contained several mitochondria and pleomorphic vesicles often clustered at the active zone (Figs. $4,5 B, 6 F$, $7 C, D, 8 C)$. The boutons form symmetrical synapses with fairly short active zones, but a single bouton may form more than one active zone with the same postsynaptic structure (Fig. $7 C$ ). The postsynaptic targets of the boutons derived from the globus pallidus included perikarya and large and small diameter dendrites in both the entopeduncular nucleus and the subthalamic nucleus. In general terms, the distributions of postsynaptic targets are consistent with previous observations (Table 1).

\section{Terminals derived from the ventral pallidum}

Terminals anterogradely labeled from the ventral pallidum displayed similar morphological features whether they were observed in the entopeduncular nucleus, lateral hypothalamus, or subthalamic nucleus. The features were similar to those of terminals derived from the dorsal aspect of the pallidal complex, the globus pallidus (see above and Smith et al., 1990; Smith and Bolam, 1991; Bolam and Smith, 1992). They were large boutons with a mean ( \pm SE) crosssectional area in the entopeduncular nucleus/lateral hypothalamic region of $0.75 \pm 0.04 \mu \mathrm{m}^{2}\left(n=71\right.$; range, $\left.0.27-1.5 \mu \mathrm{m}^{2}\right)$ and in the subthalamic nucleus/lateral hypothalamic region of $0.63 \pm 0.03 \mu \mathrm{m}^{2}$ ( $n=88$; range, $0.17-1.8 \mu \mathrm{m}^{2}$ ). They usually contained several mitochondria and clusters of pleomorphic vesicles, and they formed symmetrical synaptic contact with perikarya and both proximal and distal dendrites of neurons in each of the areas examined (Table 1). They often formed multiple contacts with their targets (Fig. 5C). As has been observed previously for terminals derived from the globus pallidus (Bolam and Smith, 1992; Bolam et al., 1993), a high proportion of terminals derived from the ventral pallidum made synaptic contact with perikarya, although most were in contact with dendrites with diameters of $<1.5 \mu \mathrm{m}$ (Table 1). Post-embedding immunolabeling revealed that the terminals derived from the ventral pallidum in the entopeduncular nucleus, the lateral hypothalamus, and the subthalamic nucleus, like those derived from the globus pallidus (Smith et al., 1990; Smith and Bolam, 1991; Bolam and Smith, 1992), were significantly ( $p<0.0005$, Mann-Whitney $\mathrm{U}$ test) enriched in GABA immunoreactivity when compared with terminals forming asymmetrical synapses in the same section (index of GABA immunoreactivity in the entopeduncular nucleus/lateral hypo- thalamus: $4.71 \pm 0.41, n=22$; in the subthalamic nucleus/ lateral hypothalamus: $16.97 \pm 1.74, n=30$ ).

\section{Synaptic convergence of terminals derived from the ventral pallidum and the globus pallidus}

The sections prepared for electron microscopic analysis, i.e., those in which BDHC and DAB were used as chromogens for the peroxidase reactions, were examined for areas of close overlap between the two sets of anterogradely labeled terminals and for individual neuronal perikarya and large proximal dendrites that were apposed by boutons derived from both the ventral pallidum and the globus pallidus. These regions were re-embedded on blocks of resin and resectioned for electron microscopy. Using this correlated light and electron microscopic approach, we examined six neurons from the entopeduncular nucleus (from two rats), two neurons from the lateral hypothalamus adjacent to the entopeduncular nucleus (from two rats), and six neurons from the subthalamic nucleus (from three rats).

In the entopeduncular nucleus, synaptic convergence of terminals derived from the ventral pallidum and those derived from the globus pallidus was observed at the level of the perikarya and proximal dendrites (Figs. 4, 5). In most cases, more than one labeled terminal from either the ventral pallidum or the globus pallidus was involved in the convergence (Fig. 4). In each of the cases, the structures postsynaptic to the anterogradely labeled terminals also received afferent synaptic input from unlabeled terminals that had the morphology of pallidal terminals, of terminals derived from the striatum (Bolam and Smith, 1992), from the subthalamic nucleus (Bevan et al., 1994b), and from the mesopontine tegmentum (Clarke et al., 1996b) (not illustrated). Of the six entopeduncular neurons reembedded for electron microscopic analysis, all were confirmed as receiving convergent synaptic input from both the ventral pallidum and the dorsal globus pallidus.

In the lateral hypothalamus, synaptic convergence of both sets of anterogradely labeled terminals was observed at the level of the perikarya and proximal dendrites in the two neurons that were examined by correlated light and electron microscopy. Both neurons were observed to receive multiple inputs from labeled terminals (Fig. 6).

In the subthalamic nucleus, terminals derived from the ventral pallidum and terminals derived from the globus pallidus made convergent synaptic contact with the same postsynaptic structure (Figs. 7, 8). Of the six neurons re-embedded for electron microscopic analysis, two were identified as receiving convergent input at the level of the perikaryon. In the four other cases, either the synaptic specializations were not observed or the terminals made synaptic contact with different structures. An additional perikaryon and distal dendrite, which were not identified at the light microscopic level before the electron microscopic analysis, were observed to receive convergent synaptic input from the ventral pallidum and the globus pallidus. The neuronal profiles receiving the convergent synaptic inputs were also postsynaptic to unlabeled terminals that had the morphological features of pallidal terminals and terminals forming asymmetrical synapses that were probably derived from the cortex, the thalamus, and the mesopontine tegmentum (Bevan and Bolam, 1995b; Bevan et al., 1995a).

\section{Afferent synaptic input to neurons of the subthalamic nucleus}

The double anterograde labeling allowed only the analysis of pallidal inputs to the perikarya and proximal regions of neu- 
Figure 5. Synaptic convergence of terminals derived from different functional domains of the pallidal complex in the entopeduncular nucleus. $A$, The large proximal dendrite of a neuron in the entopeduncular nucleus $(E P n)$ is apposed by two anterogradely labeled boutons. The bouton, shown at high power in $B$, contains the floccular DAB reaction product that adheres to the external surface of vesicle and mitochondrial membranes and indicates that it is derived from the globus pallidus $\left(b_{G P}\right)$. The bouton forms symmetrical synaptic contact (arrow) with dendrite. The other bouton contains the BDHC reaction product that is granular in appearance and is located only at restricted sites in the bouton (granules indicated by curved arrows in $C$ ), indicating that it is derived from the ventral pallidum $\left(b_{V P}\right)$. It forms multiple symmetrical contacts with the dendrite (arrows). Scale bars: $A, 5 \mu \mathrm{m} ; B, 1 \mu \mathrm{m}$.

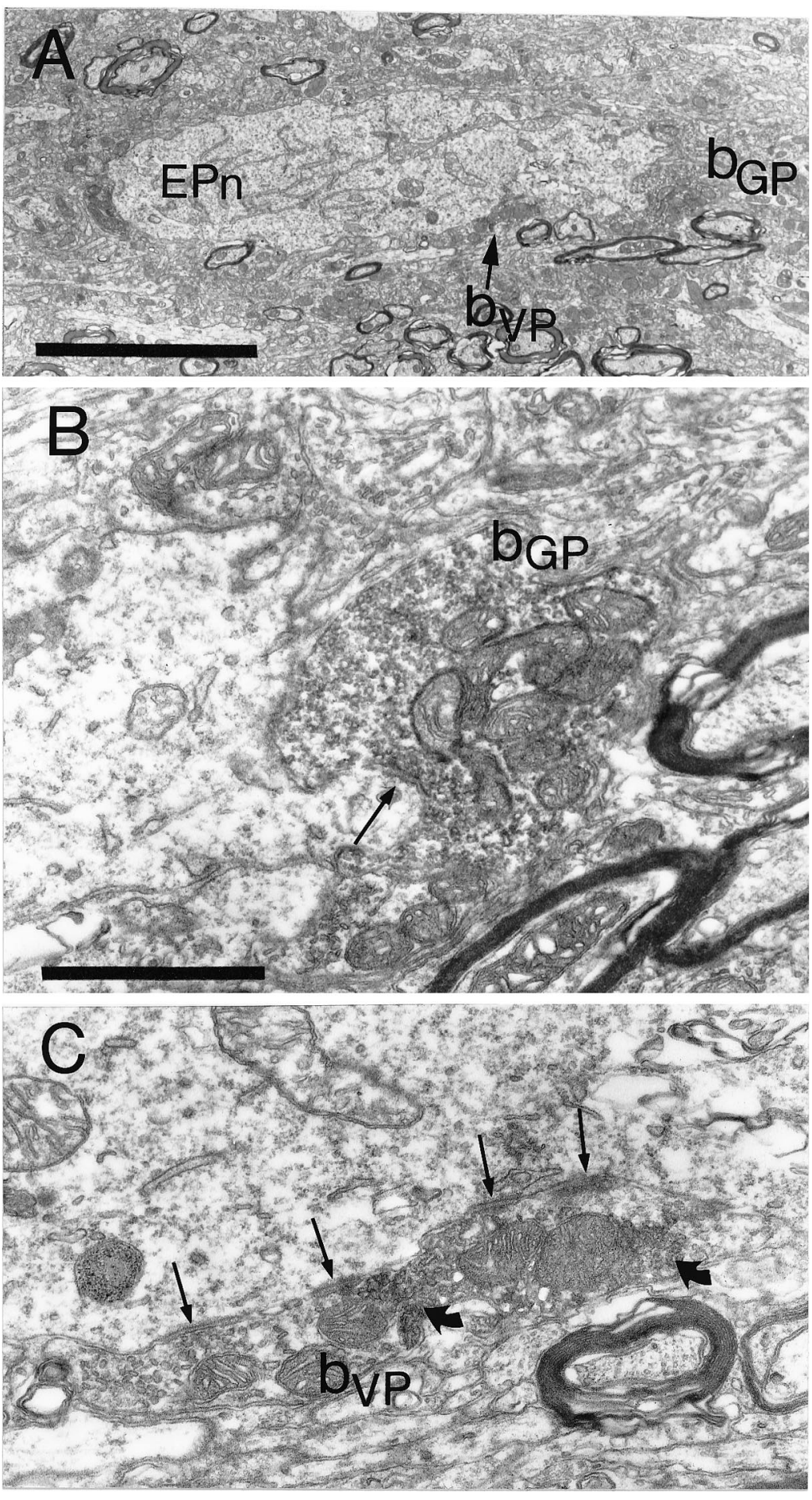

rons in the subthalamic nucleus, entopeduncular nucleus, and lateralhypothalamus. Because it is known that the dendrites of neurons of the subthalamic nucleus are oriented so that they cross functional territories defined by the pallidal input, the distal dendrites of subthalamic neurons were examined for synaptic input from pallidal terminals defined on morphological and neurochemical grounds. Five neurons in the subthalamic nucleus (from two of the injected animals) that were retrogradely labeled from the substantia nigra were examined in serial sections in the electron microscope. The neurons were selected on the basis of the extent of their dendritic labeling and the orientation and position of the dendrites in relation to the functional territories of the subthalamic nucleus. The dendrites beyond $100 \mu \mathrm{m}$ from the perikaryon were examined in the electron microscope. Pallidal terminals, defined on the basis of morphology of the bouton, the type of synaptic specialization, and the presence of relatively high levels of GABA immunoreactivity (mean \pm SEM of the index of GABA immunoreactivity, $8.96 \pm 0.72 ; n=37$ ), were found in synaptic contact with all parts of the dendritic trees examined, including distal dendrites as far as $330 \mu \mathrm{m}$ from the perikaryon (Fig. 9, Table 2). 

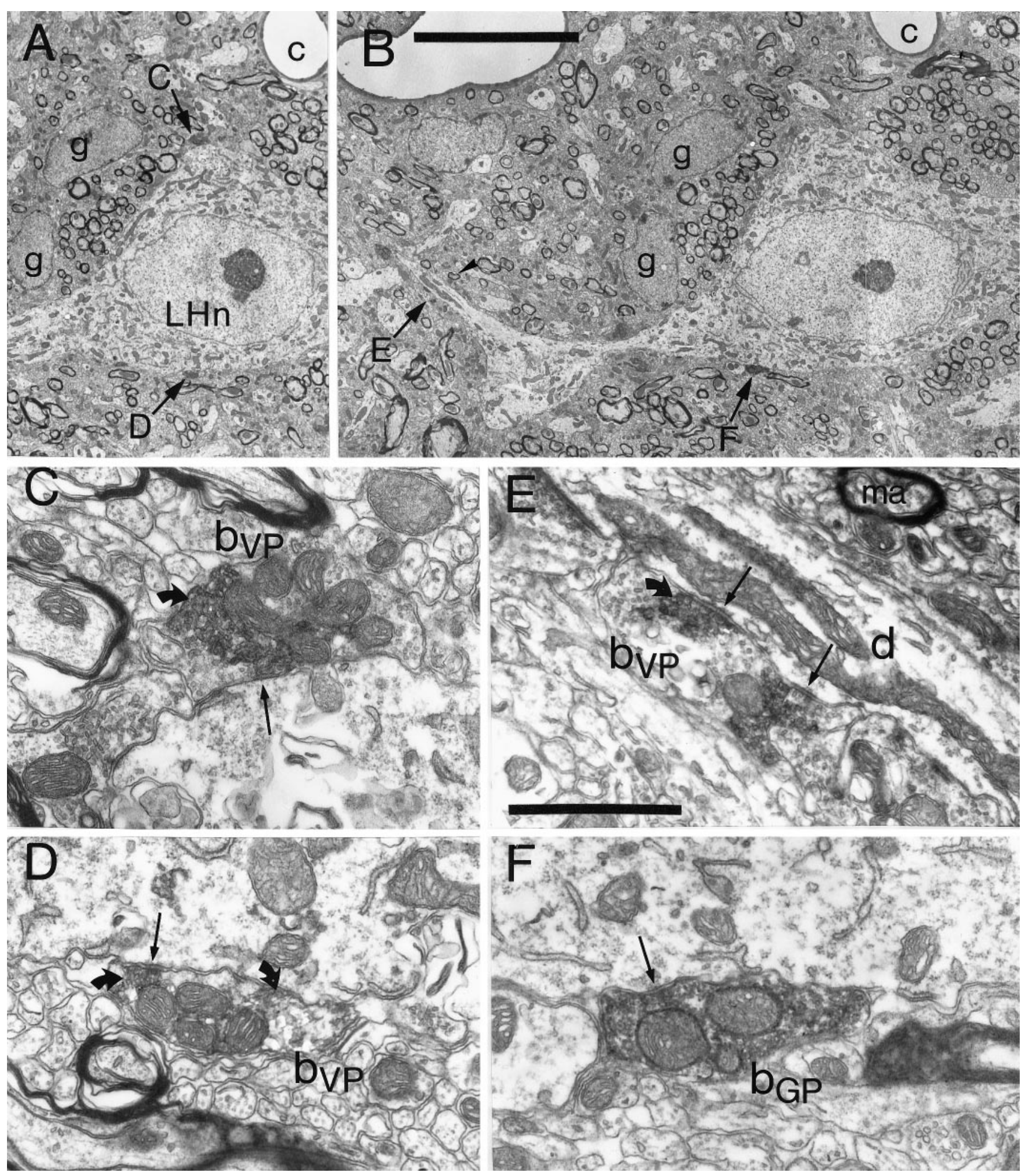

Figure 6. Synaptic convergence of terminals derived from different functional domains of the pallidal complex in the lateral hypothalamus adjacent to the entopeduncular nucleus. $A, B$, Low-power micrographs at two levels of a neuron in the lateral hypothalamus $(L H n)$ that was apposed by four anterogradely labeled terminals. Three of the terminals $\left(b_{V P}\right)$, the positions of which are indicated by arrows and the letters $C$, $D$, and $E$, are shown at higher magnification in $C, D$, and $E$, respectively. Each contains the BDHC reaction product (some granules of which are indicated by curved arrows) that was used to reveal the PHA-L transported from the ventral pallidum. The reaction product characteristically occupies only part of the labeled boutons. In each case the boutons form symmetrical synaptic contact (arrows) with the perikaryon $(C, D)$ or the dendrite $(d$ in $E)$. The micrograph $E$ is a serial section of that in $B$; the myelinated axon $(m a)$ is indicated by an arrowhead in $B$. The fourth bouton $\left(b_{G P}\right)$, the position of which is indicated by $F$ in $B$, is shown at high magnification in $F$ and contains the floccular DAB reaction product that adheres to membranes and occupies the whole of the labeled structure, identifying it as arising from the globus pallidus. This bouton also makes symmetrical synaptic contact (arrow) with the perikaryon of the lateral hypothalamic neuron. Glial cells $(g)$ and a capillary $(c)$ are labeled for correlation between the two low-power micrographs. Scale bars: $A$, $B$ (shown in $B$ ), $10 \mu \mathrm{m} ; C-E$ (shown in $E$ ), $1 \mu \mathrm{m}$. 

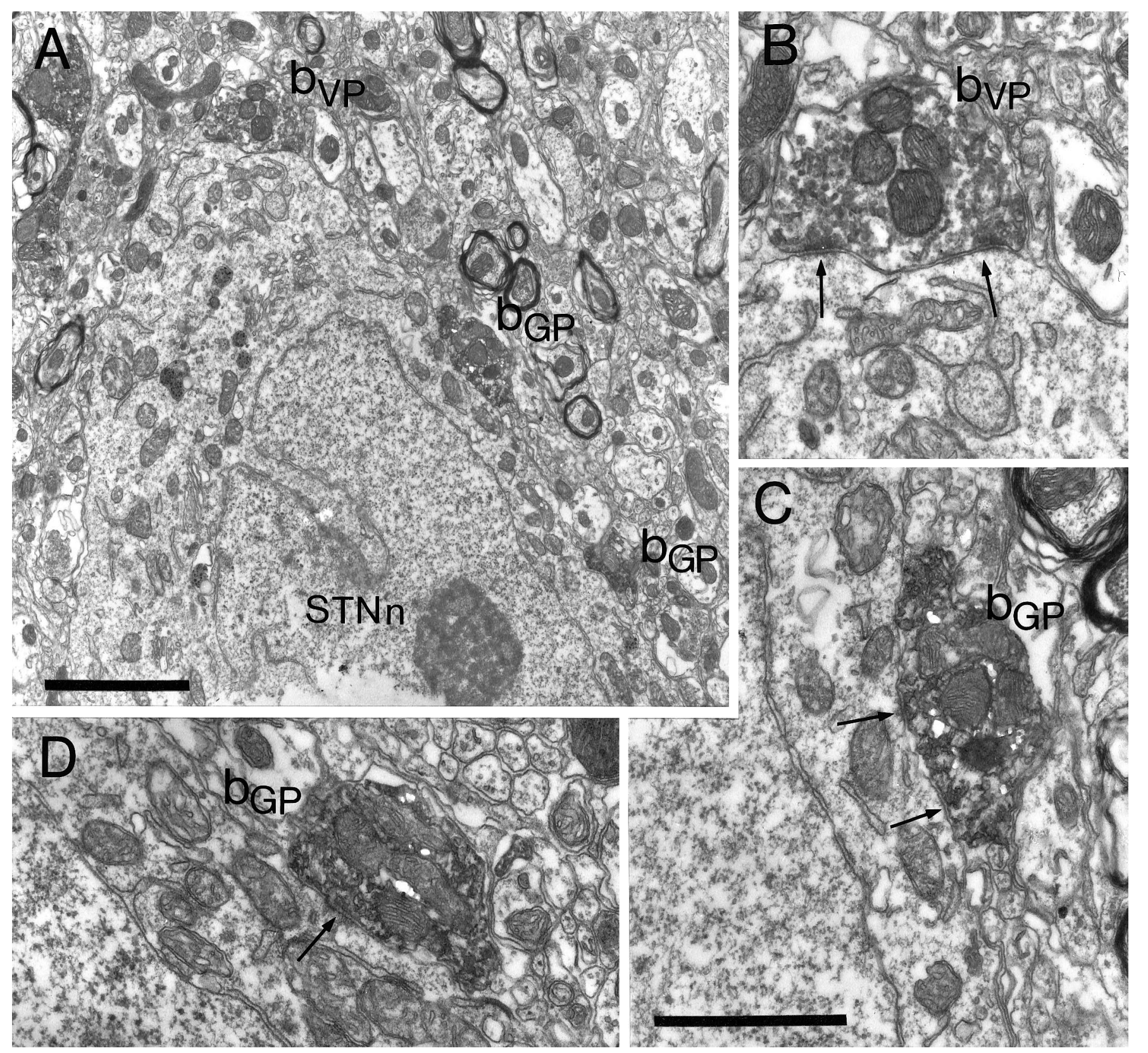

Figure 7. Synaptic convergence of terminals derived from different functional domains of the pallidal complex in the subthalamic nucleus. $A$, Part of the cell body of a neuron in the subthalamic nucleus $(S T N n)$ that is apposed by three anterogradely labeled terminals $\left(b_{V P}, b_{G P}\right)$ shown at higher magnification in $B-D$. In this animal the injections were reversed, i.e., the PHA-L was injected in and anterogradely transported from the globus pallidus, and the BDA was injected in and anterogradely transported from the ventral pallidum. One of the boutons is lightly labeled with the DAB reaction product that adheres to vesicle and mitochondrial membranes, identifying it as arising in the ventral pallidum $\left(b_{V P}\right)$. It is shown at higher magnification in $B$. The bouton forms symmetrical synaptic contacts (arrows) with the subthalamic neuron. The other two boutons, shown at high magnification in $C$ and $D$, are strongly labeled with the crystalline BDHC reaction product, which has an irregular appearance. These boutons are thus derived from the globus pallidus $\left(b_{G P}\right)$ and form symmetrical synaptic contacts with the neuron (arrows). Note that micrograph $D$ is a different serial section of that shown in $A$. Scale bars: $A$, $2 \mu \mathrm{m} ; B-D$ (shown in $C$ ), $1 \mu \mathrm{m}$.

\section{DISCUSSION}

The findings of the present study together with data from a previous study (Bevan et al., 1996) enable us to define anatomical substrates by which information carried by the descending projections of the pallidal complex may be synaptically integrated at the level of the output neurons of the basal ganglia in the substantia nigra pars reticulata and the entopeduncular nucleus, and at the level of neurons in the lateral hypothalamus and subthalamic nucleus (Fig. 10). First, our findings in the present paper confirm previous observations that functionally distinct regions of the pallidal complex provide a dense, topographic innervation of the entopeduncular nucleus and the subthalamic nucleus and their medial extensions in the lateral hypothalamus (see references cited in introductory remarks). Second, the double anterograde tracing revealed that at the interface between topographically adjacent projections, there is a zone of overlap in which individual 

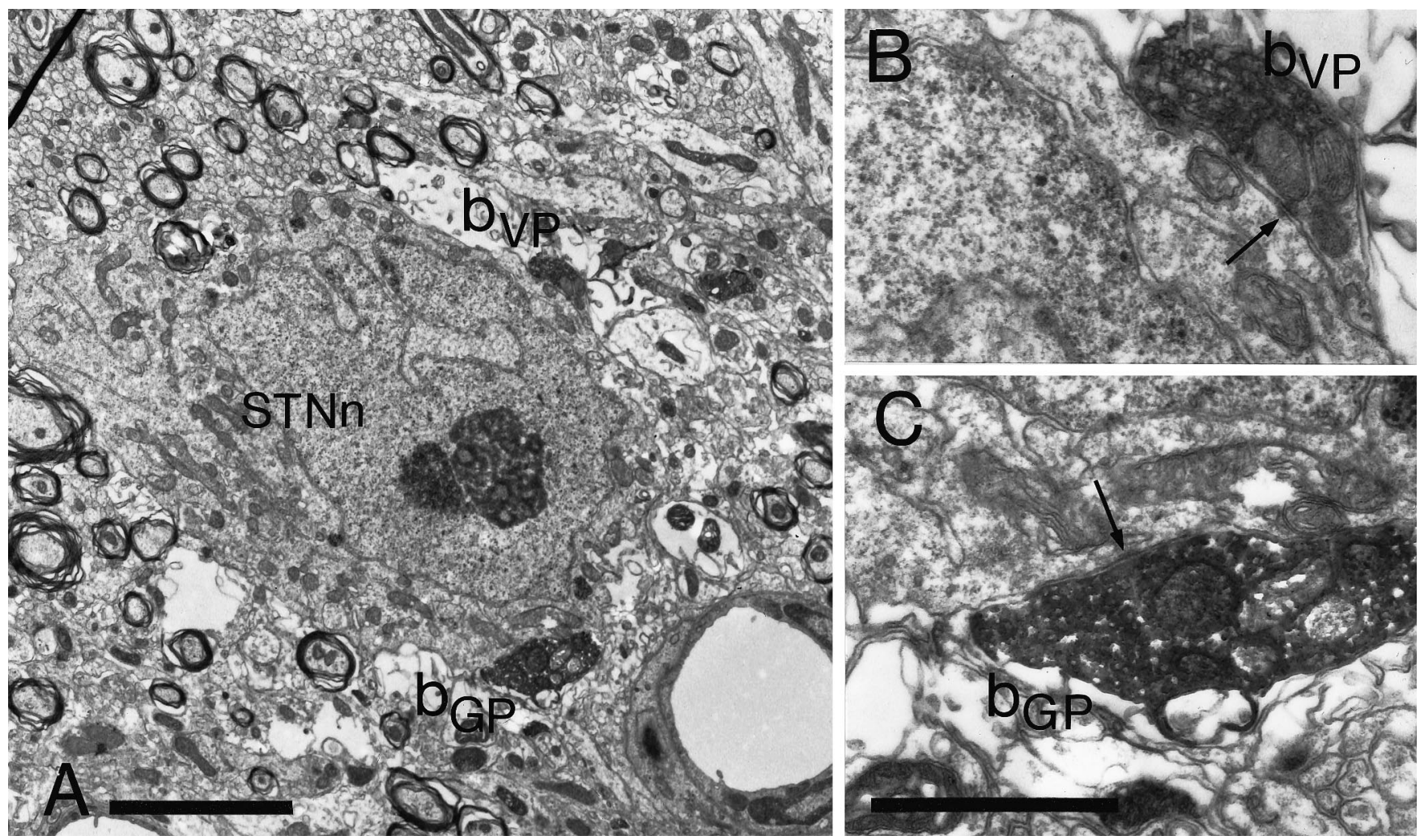

Figure 8. Synaptic convergence of terminals derived from different functional domains of the pallidal complex in the subthalamic nucleus. $A$, Cell body of a neuron in the subthalamic nucleus $(S T N n)$ that is postsynaptic to two anterogradely labeled boutons. The bouton $b_{V P}$, shown at high magnification in $B$, contains the crystalline BDHC reaction product that occupies only part of the bouton. The BDHC was used to reveal the PHA-L transported from the ventral pallidum. The bouton forms symmetrical synaptic contact (arrow) with the neuron. The bouton $b_{G P}$, shown at higher magnification in $C$, is intensely labeled with the floccular DAB reaction product used to localize the BDA transported from the globus pallidus. The reaction product fills the whole of the bouton and obscures most internal structures. This bouton forms symmetrical synaptic contact with the neuron (arrow). Scale bars: $A, 3 \mu \mathrm{m}$; $B, C$ (shown in $C$ ), $1 \mu \mathrm{m}$.

neurons are apposed by terminals derived from both the globus pallidus and the ventral pallidum. Third, neurons in the zones of overlap receive convergent synaptic input at the level of the cell body and proximal dendrites from the functionally distinct, GABAergic, pallidal projections. Fourth, as has been reported for terminals from the globus pallidus, the postsynaptic targets of terminals derived from the ventral pallidum include distal dendrites as well as perikarya and proximal dendrites (Table 1). Fifth, the orientation of dendrites and the dendritic radius of neurons in the subthalamic nucleus (also see Kita et al., 1983) is such that they cross functional boundaries within the nucleus and indeed receive synaptic input from terminals derived from the pallidal complex along their whole length (as far as $330 \mu \mathrm{m}$ from the perikaryon), indicating that convergent input also occurs at the level of dendrites (also see Shink and Smith, 1995b). A similar organization of dendrites has been reported for the entopeduncular nucleus (Nakanishi et al., 1991) and the substantia nigra pars reticulata (Grofova et al., 1982).

The present observations together with data from other tracing studies (Smith et al., 1990; Smith and Bolam, 1991; Bolam and Smith, 1992; Bolam et al., 1993; Bevan et al., 1994a,b; Smith et al., 1994; Shink and Smith, 1995a; Bevan et al., 1996; Shink et al., 1996) lead us to conclude that functionally diverse information carried by the descending projections of the pallidal complex is synaptically integrated by two mechanisms (Fig. 10). First, neurons located at the interface between functionally distinct but topographically adjacent projections integrate the diverse func- tional information at the level of the cell body and proximal dendrites. Second, the orientation and dimensions of dendrites in relation to the topography of the pallidal inputs to the subthalamic nucleus and the distribution of the pallidal inputs along the dendrites of subthalamic neurons imply that integration of pallidal information also occurs at the level of their more distal dendrites. In view of the distribution of postsynaptic targets of terminals derived from the pallidal complex in the entopeduncular nucleus and the substantia nigra pars reticulata (Table 1), and the morphology and orientation of dendrites of entopeduncular and nigral neurons, it is likely that this mode of synaptic integration of functionally diverse pallidal information also occurs in these two regions (Fig. 10). Because synaptic input to the perikaryon is likely to have a more marked effect on the activity of neurons than inputs to distal dendrites, the pallidal input to the region in which the perikaryon of the target neuron lies may have a greater influence on the activity of the neuron than the pallidal input to the distal dendrites, although their pattern of activity and the distribution and types of GABA receptors on these neurons will also be a factors.

The conclusions of our studies are dependent on two issues: first, the technical issues relating to the use of double anterograde labeling techniques at both the light and electron microscopic levels. These issues have been discussed extensively on previous occasions and so will not be dealt with here (Smith and Bolam, 1991, 1992; Bolam and Smith, 1992; Bolam et al., 1993). The second issue relates to the functionality of the regions of the pallidal complex that we injected. 
It is well recognized that the functionality of different regions of the pallidal complex is dependent on its input from the striatal complex and ultimately on the source of innervation of that region of the striatal complex. Thus the regions of the pallidal complex that we injected, the ventral pallidum and the globus pallidus, receive their major input from the nucleus accumbens and the dorsal striatum, respectively. The nucleus accumbens in turn receives major inputs from limbic regions of the brain, including the hippocampus and the amygdala, whereas the major input to the dorsal striatum is from motor and associative regions of the cortex (Kunzle, 1975; Goldman and Nauta, 1977; Van Hoesen et al., 1981; Nauta and Domesick, 1984; Selemon and Goldman-Rakic, 1985; Alexander et al., 1986, 1990; McGeorge and Faull, 1989; Flaherty and Graybiel, 1991; Hoover and Strick, 1993; Groenewegen and Berendse, 1994; Joel and Weiner, 1994). Thus the ventral pallidum is recognized as the limbic division of the pallidal complex, and the globus pallidus as the motor and associative division. We ensured that our deposits of tracers were confined to either the ventral pallidum or the globus pallidus by comparing the location of the deposits with the region of dense substance $\mathrm{P}$ immunoreactivity, which is a marker for the ventral pallidum (Haber and Nauta, 1983; Groenewegen et al., 1993), and by comparing the distribution of neurons in the striatal complex that became retrogradely labeled after the tracer deposits in the pallidal complex (Fig. 3). The latter observation not only confirmed that the location of the deposits lay within the ventral pallidum or the globus pallidus, but also confirmed that the areas that received the deposits were probably functionally distinct in that they received input from the nucleus accumbens and the dorsal striatum, respectively.

\section{Functional considerations}

An issue that has been the subject of much debate is the nature of information processing by the basal ganglia. Concepts of multiple, parallel, functionally segregated pathways of information-flow through the basal ganglia, as opposed to concepts of convergence and integration, have been proposed (Francois et al., 1984; Percheron et al., 1984, 1987; Alexander et al., 1986, 1990; Flaherty and Graybiel, 1991, 1993, 1994; Percheron and Filion, 1991; Parthasarathy et al., 1992; Hoover and Strick, 1993; Groenewegen and Berendse, 1994; Joel and Weiner, 1994; Lynch et al., 1994). From tracing studies it is evident that there is a functional topography that is maintained throughout the cortico-basal gangliathalamocortical system; however, it is also clear from behavioral and physiological analyses and from clinical data that one of the functions of the basal ganglia is to integrate diverse information in the production of context-dependent behavior (Wurtz and Hikosaka, 1986; Schultz et al., 1993; Graybiel et al., 1994; Aosaki et al., 1995). Most authors agree that synaptic convergence does occur, and the number of neurons in each nucleus of the basal ganglia (Oorschot, 1996) indicates that there is a high degree of convergence in the system we have analyzed and in the basal ganglia in general. Such convergence could be between neurons at each level

\begin{tabular}{|c|c|c|c|c|c|}
\hline \multirow{2}{*}{$\begin{array}{l}\text { Neuron } \\
\text { number }\end{array}$} & \multicolumn{5}{|c|}{$\begin{array}{l}\text { Location and numbers of synaptic boutons } \\
\text { ( } \mu \mathrm{m} \text { from cell body) }\end{array}$} \\
\hline & $100-150$ & $150-200$ & $200-250$ & $250-300$ & $300+$ \\
\hline 1 & 4 & & & & \\
\hline 2 & 3 & 4 & & & \\
\hline 3 & 0 & 2 & 3 & & \\
\hline 4 & 0 & 0 & 7 & 5 & 1 \\
\hline 5 & 4 & 1 & 4 & 3 & \\
\hline Total & 11 & 7 & 14 & 8 & 1 \\
\hline
\end{tabular}

For each neuron, a single dendrite was serially sectioned, and the number of pallidal terminals was identified on the basis of morphological features, type of synaptic specialization, and in most cases $(35 / 41)$, the presence of GABA immunoreactivity. The analysis was commenced at $100 \mu \mathrm{m}$ from the perikaryon and continued as far as the dendritic staining extended or until it left the section. For each neuron the last figure represents the maximum extent.

within one of the functionally distinct parallel pathways that may mediate the convergence of information related in different ways to the common goal of controlling a particular movement, behavior, or cognitive process. Indeed, the elegant studies of Graybiel and colleagues (Flaherty and Graybiel, 1991, 1993, 1994; Parthasarathy et al., 1992) show clearly that in the corticostriatal system, groups of neurons in motor and somatosensory cortices that are related to a particular body part provide overlapping (and presumably synaptic convergent) projections to specific regions of the neostriatum. It has also been suggested, however, that the organization of the striatopallidal system and the organization of dendrites in the globus pallidus provide a substrate for synaptic integration of widely diverse functional information arising from the neostriatum (Francois et al., 1984; Percheron et al., 1984, 1987; Percheron and Filion, 1991), although there is no evidence as yet at the synaptic level. The ascending dopaminergic system may underlie a similar role (Somogyi et al., 1981; Nauta and Domesick, 1984; Gerfen et al., 1987; Jimenez-Castellanos and Graybiel, 1987; Smith and Bolam, 1990). Our data provide the first direct evidence of an anatomical substrate at the synaptic level for the convergence of functionally diverse information by a descending projection system in the basal ganglia. It is unclear at present whether within this system or as part of this system there is also synaptic convergence of small groups of neurons related in different ways to a common goal of controlling a particular process.

An important principle emerges from the topographical organization of the descending projection of the pallidal complex (Alexander et al., 1986, 1990; Smith and Bolam, 1989, 1991; Groenewegen et al., 1993; Hoover and Strick, 1993; Groenewegen and Berendse, 1994; Joel and Weiner, 1994; Lynch et al., 1994). Convergent pallidal input to the proximal regions of neurons in the subthalamic nucleus (and other nuclei of the basal ganglia)

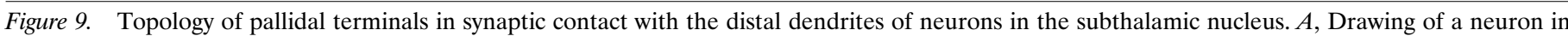

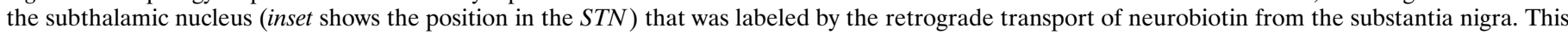

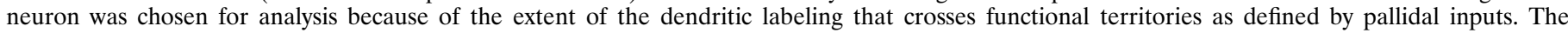

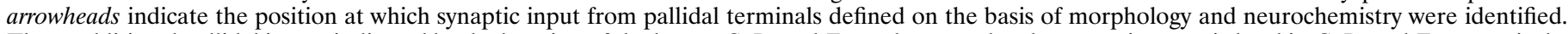

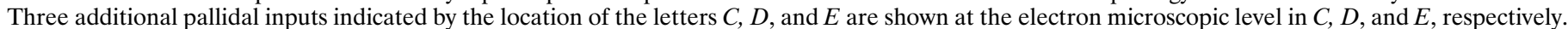

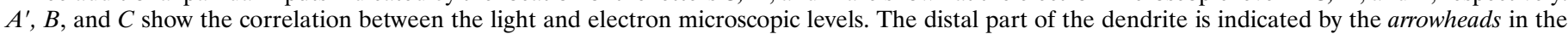

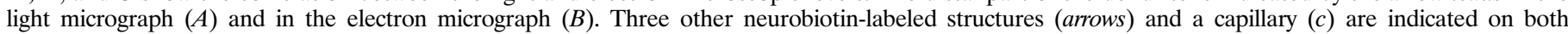

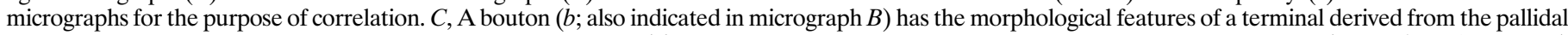

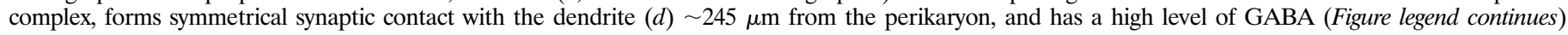



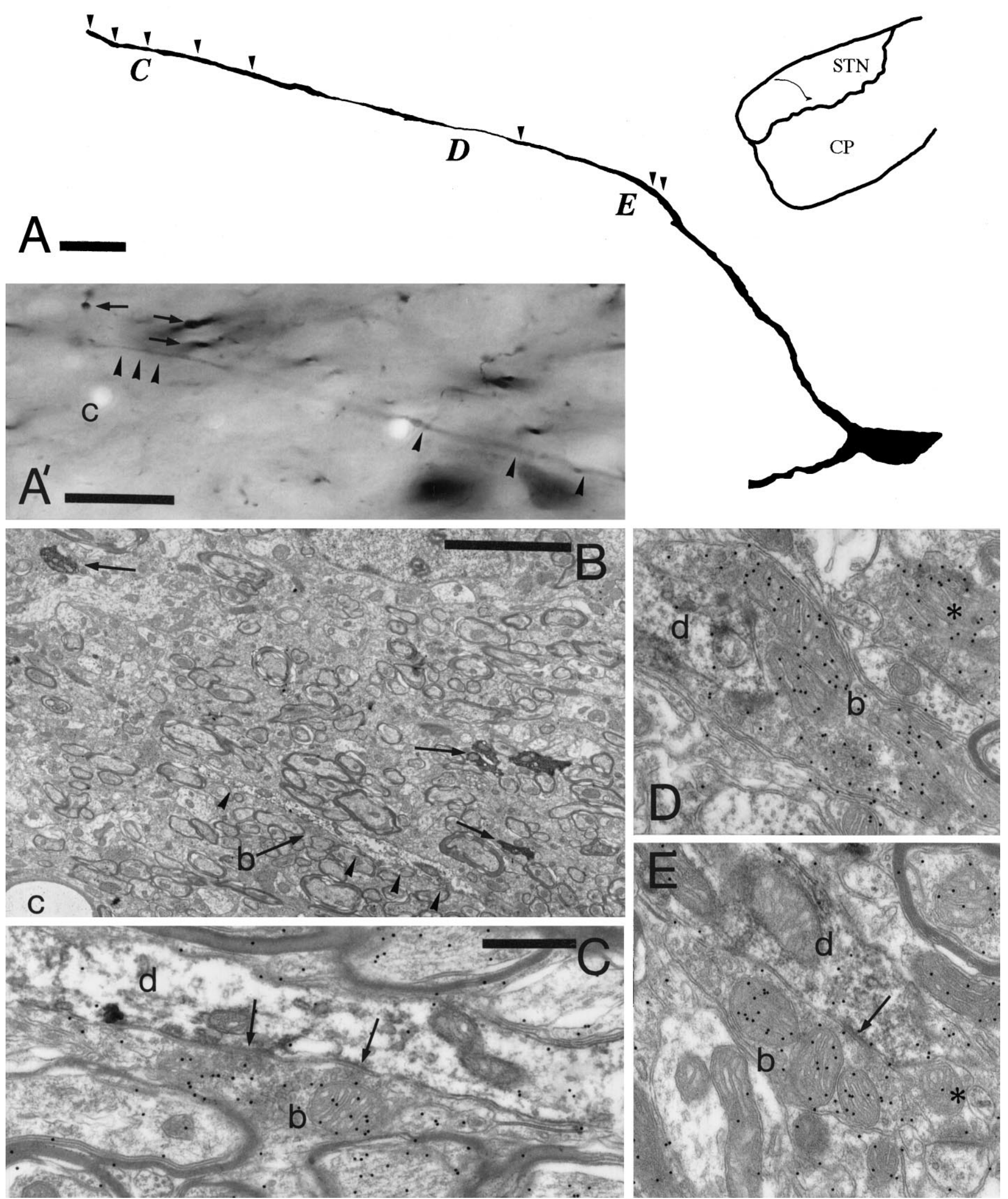

immunoreactivity associated with it (index of GABA immunoreactivity is 5.8 compared with GABA-negative terminals forming asymmetrical synapses in the same section). The bouton $(b)$ in $D$ also possesses the features of a pallidal terminal, is $\sim 145 \mu \mathrm{m}$ from the perikaryon, and has an index of GABA immunoreactivity of 11.2. In $E$ the bouton $(b)$ forms symmetrical synaptic contact with the dendrite (arrow) $\sim 100 \mu \mathrm{m}$ from the perikaryon and has an index of GABA immunoreactivity of 6.54. The asterisk in $D$ indicates a bouton that forms symmetrical synaptic contact with a dendrite and has an index of GABA immunoreactivity of 9.49. The asterisk in $E$ indicates a terminal that forms an asymmetrical synapse with a spine and is GABA-negative (index of GABA immunoreactivity 1.19). Scale bars: $A, 20 \mu \mathrm{m} ; A^{\prime}, 20 \mu \mathrm{m} ; B, 5 \mu \mathrm{m} ; C-E$ (shown in $C$ ), $0.5 \mu \mathrm{m}$. 


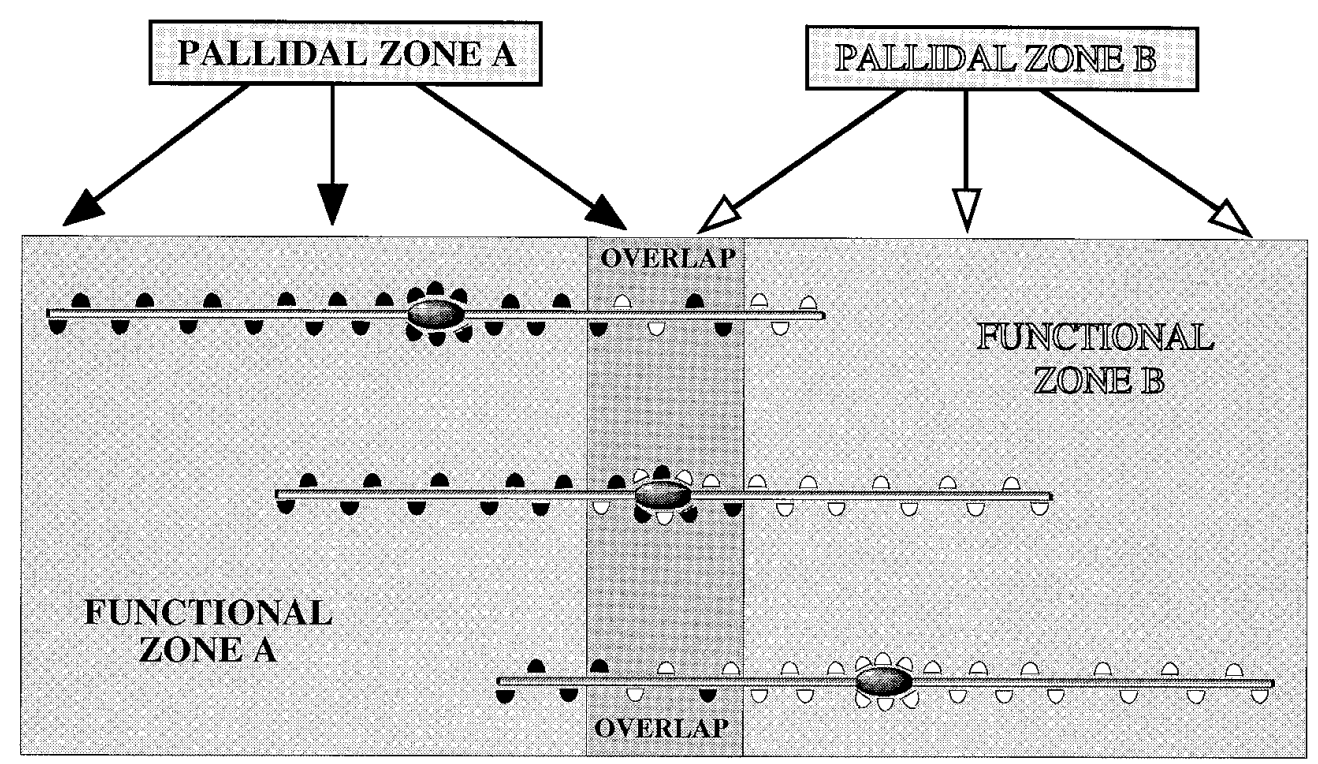

Figure 10. Schematic summary of the somatic and dendritic modes of synaptic integration of descending, functionally distinct pallidal projections in the subthalamic nucleus revealed by double anterograde labeling and electron microscopy. The pallidal complex provides projections to the subthalamic nucleus that largely maintain the functional topography. Adjacent populations of neurons, illustrated by PALLIDAL ZONE A and PALLIDAL ZONE $B$ and giving rise to black and white boutons, respectively, although mainly innervating separate but adjacent regions of the subthalamic nucleus, also give rise to a region of overlap. The dimensions and orientation of dendrites of a large proportion of neurons in the subthalamic nucleus are such that they extend in a mediolateral or ventrodorsal direction and thus cross the functional boundaries defined by the pallidal inputs. Integration of the descending, functionally diverse information from the pallidal complex occurs by synaptic convergence on individual neurons in the subthalamic nucleus. This is mediated by two systems: synaptic integration at the level of the soma and proximal dendrites, and synaptic integration at the level of more distal dendrites. Synaptic integration of pallidal inputs occurs at the level of the soma and proximal dendrites of neurons located in the region of overlap of the pallidal projections (middle neuron). These neurons receive synaptic input to their cell bodies and proximal dendrites from neurons located in both pallidal zones. Their distal dendrites, if oriented across the boundaries, will also receive inputs from both pallidal zones. The net weights of the two pallidal inputs to these neurons are likely to be similar, although the distal dendrites will preferentially receive inputs from one or the other of the pallidal zones. The second mode of synaptic integration occurs on the distal dendrites of neurons, the cell bodies of which are located within a functional zone defined on the basis of its pallidal input (top and bottom neurons in the diagram). A high proportion of the pallidal input to these neurons is derived from the single pallidal zone (PALLIDAL ZONE $A$ or $B$ ) that provides the innervation of the functional zone (FUNCTIONAL ZONE $A$ or $B$ ) within which they reside. The dendrites that cross functional boundaries, however, receive synaptic input from the topographically adjacent pallidal zone in the region of overlap and in the adjacent functional zone of the subthalamic nucleus. In this case the pallidal input will be weighted in favor of the pallidal region that projects to the zone within which the neuron (mainly) resides, but will vary with the position of the neurons in relation to the boundaries and the number of dendrites crossing the boundary. In this system also, it is possible that individual dendrites will receive inputs from a single pallidal zone. The data on which this model is based were derived from injections of tracers into broad functional zones of the pallidal complex, i.e., the limbic division (ventral pallidum) and the motor and associative division (globus pallidus) that nevertheless give rise to adjacent projections. Topographic studies suggest that these broad functional zones are themselves subdivided into small groups of functionally related neurons that are organized into multiple parallel pathways. We predict that the same principles of organization will apply to these subdivisions of the pallidal complex. Although the present model is based mostly on findings of neurons in the subthalamic nucleus, the present work and previously published data (Bevan et al., 1996) indicate that the somatic integration in areas of overlap of pallidal projections also occurs in the entopeduncular nucleus, the lateral hypothalamus, and the substantia nigra pars reticulata. Furthermore, the synaptology of the pallidal projections and the synaptology and orientation of dendrites in these regions indicate that integration also probably occurs at the level of distal dendrites (see text for references). Although synaptic convergence of functionally diverse pallidal inputs also occurs on dopamine neurons of the substantia nigra pars compacta and the ventral tegmental area (Bevan et al., 1996), the exact rules of synaptic integration remain to be established.

probably arises from pallidal neurons that are closely spaced and presumably more closely related in functional terms. Convergent input to more distal dendrites probably arises from pallidal neurons that are more widely spaced and therefore presumably more widely separated in functional terms. It remains to be established whether pallidal inputs located on different parts of the soma and dendritic tree have different effects on the firing or activity of the postsynaptic neuron. The findings of pallidal inputs to the distal dendrites of neurons in the subthalamic nucleus and the fact that the orientation of their dendrites are such that they cross functional boundaries defined by the pallidal input implies that convergence of functionally diverse information occurs more commonly than can be surmised from the degree of overlap of the projections.

The precise role of neurons of the pallidal complex remains unclear. They are part of the neuronal network of the so-called "indirect pathway" and as such are involved in the resting inhibitory output of the basal ganglia and presumably the periods of inhibition of basal ganglia targets that occur during complex behaviors (Albin et al., 1989; Alexander and Crutcher, 1990; DeLong, 1990; Gerfen and Wilson, 1996; Shink et al., 1996). Our data indicate that activity in the indirect pathway is probably important in the integration of functionally diverse information. The parallel but distributed and partly convergent network of the descending projections of the pallidal complex provides an anatomical basis for the association of functionally diverse information that may be of importance in the production of integrated behavior and learning.

\section{Concluding remarks}

Our confirmation of the topography of the descending projections of the pallidal complex and the findings of synaptic convergence 
reconcile the two opposing views of segregation and integration. The findings emphasize the point that when considering the topographical and functional organization of the basal ganglia, we must consider not only the topography of projections but also the arrangement of dendrites and the synaptic organization of both the projection axons and the neurons in the target regions. Our results and those of others demonstrate that within the basal ganglia, systems for parallel channels, systems for the synaptic integration of closely related information, and systems for the synaptic integration of functionally diverse information coexist.

\section{REFERENCES}

Albin RL, Young AB, Penney JB (1989) The functional anatomy of basal ganglia disorders. Trends Neurosci 12:366-375.

Alexander GE, Crutcher MD (1990) Functional architecture of basal ganglia circuits: neural substrates of parallel processing. Trends Neurosci $13: 266-271$

Alexander GE, DeLong MR, Strick PL (1986) Parallel organization of functionally segregated circuits linking basal ganglia and cortex. Annu Rev Neurosci 9:357-381.

Alexander GE, Crutcher MD, DeLong MR (1990) Basal gangliathalamocortical circuits: parallel substrates for motor, oculomotor, "prefrontal" and "limbic" functions. In: Prefrontal cortex. Its structure, function and pathology (Uylings HBM, Vaneden CG, Debruin JPC, Corner MA, Feenstra MGP, eds), pp 119-146. Amsterdam: Elsevier.

Aosaki T, Kimura M, Graybiel AM (1995) Temporal and spatial characteristics of tonically active neurons of the primate's striatum. J Neurophysiol 73:1234-1252.

Bevan MD, Bolam JP (1995a) Descending projections of the globus pallidus and the ventral pallidum in the rat: evidence for the convergence of functionally different pathways onto individual neurons in the basal ganglia. Brain Res Assoc Abstr 12:39.

Bevan MD, Bolam JP (1995b) Cholinergic, GABAergic and glutamateenriched inputs from the mesopontine tegmentum to the subthalamic nucleus in monkeys. J Neurosci 15:7105-7120.

Bevan MD, Bolam JP, Crossman AR (1994a) Convergent synaptic input from the neostriatum and the subthalamus onto identified nigrothalamic neurons in the rat. Eur J Neurosci 6:320-334.

Bevan MD, Crossman AR, Bolam JP (1994b) Neurons projecting from the entopeduncular nucleus to the thalamus receive convergent synaptic inputs from the subthalamic nucleus and the neostriatum. Brain Res 659:99-109.

Bevan MD, Francis CM, Bolam JP (1995a) The glutamate-enriched cortical and thalamic input to neurons in the subthalamic nucleus of the rat: convergence with GABA-positive terminals. J Comp Neurol 361:491-511.

Bevan MD, Smith AD, Bolam JP (1995b) Descending projections of the globus pallidus and the ventral pallidum in the rat: convergence of functionally different pathways onto individual neurons in the basal ganglia. Soc Neurosci Abstr 21:1906.

Bevan MD, Smith AD, Bolam JP (1996) The substantia nigra as a site of synaptic integration of functionally diverse information arising from the ventral pallidum and the globus pallidus in the rat. Neuroscience 75:5-12.

Bolam JP, Bennett B (1995) The microcircuitry of the neostriatum. In: Molecular and cellular mechanisms of neostriatal functions (Ariano M, Surmeier DJ, eds), pp 1-19. Austin, TX: R.G Landes.

Bolam JP, Smith Y (1992) The striatum and the globus pallidus send convergent synaptic inputs onto single cells in the entopeduncular nucleus of the rat: a double anterograde labeling study combined with post-embedding immunocytochemistry for GABA. J Comp Neurol 321:456-476.

Bolam JP, Smith Y, Ingham CA, von Krosigk M, Smith AD (1993) Convergence of synaptic terminals from the striatum and the globus pallidus onto single neurones in the substantia nigra and the entopeduncular nucleus. Prog Brain Res 99:73-88.

Chesselet MF, Graybiel AM (1986) Striatal neurons expressing somatostatin-like immunoreactivity: evidence for a peptidergic interneuronal system in the cat. Neuroscience 17:547-571.

Clarke NP, Bolam JP, Bevan MD (1996a) Segregation and convergence of dorsal and ventral pallidal innervation of the entopeduncular nucleus (EP) and subthalamic nucleus (STN). Brain Res Assoc Abstr 13:69.
Clarke NP, Bolam JP, Bevan MD (1996b) Glutamate-enriched inputs from the mesopontine tegmentum to the entopeduncular nucleus in the rat. Eur J Neurosci 8:1363-1376.

Cuello AC, Galfre G, Milstein C (1979) Detection in substance P in the central nervous system by a monoclonal antibody. Proc Natl Acad Sci USA 76:3532-3536.

DeLong MR (1990) Primate models of movement disorders of basal ganglia origin. Trends Neurosci 13:281-285.

Flaherty AW, Graybiel AM (1991) Corticostriatal transformations in the primate somatosensory system: projections from physiologically mapped body-part representations. J Neurophysiol 66:1249-1263.

Flaherty AW, Graybiel AM (1993) Two input systems for body representations in the primate striatal matrix: experimental evidence in the squirrel monkey. J Neurosci 13:1120-1137.

Flaherty AW, Graybiel AM (1994) Input-output organization of the sensorimotor striatum in the squirrel monkey. J Neurosci 14:599-610.

Francois C, Percheron G, Yelnik J, Heyner S (1984) A Golgi analysis of the primate globus pallidus. I. Inconstant processes of large neurons, other neuronal types, and afferent axons. J Comp Neurol 227:182-199.

Gerfen CR (1984) The neostriatal mosaic: compartmentalization of corticostriatal input and striatonigral output systems. Nature 311:461-464.

Gerfen CR, Sawchenko PE (1984) An anterograde neuroanatomical tracing method that shows the detailed morphology of neurons, their axons and terminals: immunohistochemical localization of an axonally transported plant lectin, Phaseolus vulgaris leucoagglutinin (PHA-L). Brain Res 290:219-238.

Gerfen CR, Wilson CJ (1996) The basal ganglia. In: Integrated systems of the CNS, Part III, Handbook of chemical neuroanatomy (Björklund A, Hökfelt T, Swanson L, eds), pp 369-466. Amsterdam: Elsevier.

Gerfen CR, Herkenham M, Thibault J (1987) The neostriatal mosaic. II. Patch- and matrix-directed mesostriatal dopaminergic and nondopaminergic systems. J Neurosci 7:3915-3934.

Goldman PS, Nauta WJH (1977) An intricately patterned prefrontocaudate projection in the rhesus monkey. J Comp Neurol 171:369-374.

Graybiel AM (1995) Building action repertoires: memory and learning functions of the basal ganglia. Curr Opin Neurobiol 5:733-741.

Graybiel AM, Aosaki T, Flaherty AW, Kimura M (1994) The basal ganglia and adaptive motor control. Science 265:1826-1831.

Groenewegen HJ, Berendse HW (1994) Anatomical relationships between the prefrontal cortex and the basal ganglia in the rat. In: Motor and cognitive functions of the prefrontal cortex. (Thierry A-M, Glowinski J, Goldman-Rakic PS, Christen Y, eds), pp 52-76. Berlin: Springer.

Groenewegen HJ, Berendse HW, Haber SN (1993) Organization of the output of the ventral striatopallidal system in the rat: ventral pallidal efferents. Neuroscience 57:113-142.

Grofova I, Deniau JM, Kitai ST (1982) Morphology of the substantia nigra pars reticulata projection neurons intracellularly labeled with HRP. J Comp Neurol 208:352-368.

Haber SN, Nauta WJH (1983) Ramifications of the globus pallidus in the rat as indicated by patterns of immunohistochemistry. Neuroscience 9:245-260.

Haber SN, Groenewegen HJ, Grove EA, Nauta WJH (1985) Efferent connections of the ventral pallidum: evidence of a dual striato pallidofugal pathway. J Comp Neurol 235:322-335.

Hodgson AJ, Penke B, Erdei A, Chubb IW, Somogyi P (1985) Antisera to $\gamma$-aminobutyric acid. I. Production and characterization using a new model system. J Histochem Cytochem 33:229-239.

Hoover JE, Strick PL (1993) Multiple output channels in the basal ganglia. Science 259:819-821.

Jimenez-Castellanos J, Graybiel AM (1987) Subdivisions of the dopamine-containing A8-A9-A10 complex identified by their differential mesostriatal innervation of striosomes and extrastriosomal matrix. Neuroscience 23:223-242.

Joel D, Weiner I (1994) The organization of the basal gangliathalamocortical circuits: open interconnected rather than closed segregated. Neuroscience 63:363-379.

Kincaid AE, Penney JB, Young AB, Newman SW (1991) Evidence for a projection from the globus pallidus to the entopeduncular nucleus in the rat. Neurosci Lett 128:121-125.

Kita H, Armstrong W (1991) A biotin-containing compound $\mathrm{N}$-(2aminoethyl) biotinamide for intracellular labeling and neuronal tracing studies: comparison with biocytin. J Neurosci Methods 37:141-150. 
Kita H, Chang HT, Kitai ST (1983) The morphology of intracellularly labeled rat subthalamic neurons: a light microscopic analysis. J Comp Neurol 215:245-57.

Kubota Y, Kawaguchi Y (1993) Spatial distribution of chemically identified intrinsic neurons in relation to patch and matrix compartments of rat neostriatum. J Comp Neurol 332:499-513.

Kunzle H (1975) Bilateral projections from precentral motor cortex to the putamen and other parts of the basal ganglia: an autoradiographic study in Macaca fascicularis. Brain Res 88:195-209.

Lapper SR, Bolam JP (1991) The anterograde and retrograde transport of neurobiotin in the central nervous system of the rat. J Neurosci Methods 39:163-174.

Levey AI, Bolam JP, Rye DB, Hallanger A, Demuth RM, Mesulam M-M, Wainer BH (1986) A light and electron microscopic procedure for sequential double antigen localization using diaminobenzidine and benzidine dihydrochloride. J Histochem Cytochem 34:1449-1457.

Lynch JC, Hoover JE, Strick PL (1994) Input to the primate frontal eye field from the substantia nigra, superior colliculus, and dentate nucleus demonstrated by transneuronal transport. Exp Brain Res 100:181-186.

McGeorge AJ, Faull RL (1989) The organization of the projection from the cerebral cortex to the striatum in the rat. Neuroscience 29:503-537.

Nakanishi H, Kita H, Kitai ST (1991) Intracellular study of rat entopeduncular nucleus neurons in an in vitro slice preparation: response to subthalamic stimulation. Brain Res 549:285-291.

Nauta WJH, Domesick VB (1984) Afferent and efferent relationships of the basal ganglia. In: Functions of the basal ganglia. Ciba Foundation Symposium 107 (Evered D, O'Connor M, eds), pp 3-23. London: Pitman.

Oorschot DE (1996) Total number of neurons in the neostriatal, pallidal, subthalamic, and substantia nigral nuclei of the rat basal ganglia: a stereological study using the cavalieri and optical dissector methods. J Comp Neurol 366:580-599.

Parthasarathy HB, Schall JD, Graybiel AM (1992) Distributed but convergent ordering of corticostriatal projections: analysis of the frontal eye field and the supplementary eye field in the macaque monkey. J Neurosci 12:4468-4488.

Paxinos G, Watson C (1986) The rat brain in stereotaxic coordinates, 2nd Edition. New York: Academic.

Percheron G, Filion M (1991) Parallel processing in the basal ganglia: up to a point. Trends Neurosci 14:55-59.

Percheron G, Yelnik J, Francois C (1984) A Golgi analysis of the primate globus pallidus. III. Spatial organization of the striato-pallidal complex. J Comp Neurol 227:214-227.

Percheron G, Francois C, Yelnik J (1987) Spatial organisation and information processing in the core of the basal ganglia. In: The basal ganglia II (Carpenter MB, Jayaraman A, eds), pp 205-225. New York: Plenum.

Phend KD, Weinberg RJ, Rustioni A (1992) Techniques to optimize post-embedding single and double staining for amino acid neurotransmitters. J Histochem Cytochem 40:1011-1020.

Schultz W, Apicella P, Ljungberg T (1993) Responses of monkey dopamine neurons to reward and conditioned stimuli during successive steps of learning a delayed response task. J Neurosci 13:894-899.

Selemon LD, Goldman-Rakic PS (1985) Longitudinal topography and interdigitation of corticostriatal projections in the rhesus monkey. J Neurosci 5:776-794.

Shink E, Smith Y (1995a) Differential synaptic innervation of neurons in the internal and external segments of the globus pallidus by the GABAand glutamate-containing terminals in the squirrel monkey. J Comp Neurol 358:119-141.
Shink E, Smith Y (1995b) Synaptic organisation of GABAergic terminals in the subthalamic nucleus (STN) of the squirrel monkey. Soc Neurosci Abstr 21:676.

Shink E, Bevan MD, Bolam JP, Smith Y (1996) The subthalamic nucleus and the external pallidum: two tightly interconnected structures that control the output of the basal ganglia in the monkey. Neuroscience 73:335-357.

Smith AD, Bolam JP (1990) The neural network of the basal ganglia as revealed by the study of synaptic connections of identified neurones. Trends Neurosci 13:259-265.

Smith Y, Bolam JP (1989) Neurons of the substantia nigra reticulata receive a dense GABA-containing input from the globus pallidus in the rat. Brain Res 493:160-167.

Smith Y, Bolam JP (1991) Convergence of synaptic inputs from the striatum and the globus pallidus onto identified nigrocollicular cells in the rat: a double anterograde labelling study. Neuroscience 44:45-73.

Smith Y, Bolam JP (1992) Combined approaches to experimental neuroanatomy: combined tracing and immunohistochemical techniques for the study of neuronal microcircuits. In: Experimental neuroanatomy: A practical approach (Bolam JP, ed) pp 239-266. Oxford: Oxford UP.

Smith Y, Shink E (1995) The pedunculopontine nucleus (PPN): a potential target for the convergence of information arising from different functional territories of the internal pallidum in primates. Soc Neurosci Abstr 21:677.

Smith Y, Bolam JP, von Krosigk M (1990) Topographical and synaptic organization of the GABA-containing pallidosubthalamic projection in the rat. Eur J Neurosci 2:500-511.

Smith Y, Wichmann T, DeLong MR (1994) Synaptic innervation of neurones in the internal pallidal segment by the subthalamic nucleus and the external pallidum in monkeys. J Comp Neurol 343:297-318.

Somogyi P, Hodgson AJ (1985) Antisera to $\gamma$-aminobutyric acid. III. Demonstration of GABA in Golgi-impregnated neurons and in conventional electron microscopic sections of cat striate cortex. J Histochem Cytochem 33:249-257.

Somogyi P, Bolam JP, Totterdell S, Smith AD (1981) Monosynaptic input from the nucleus accumbens-ventral striatum region to retrogradely labelled nigrostriatal neurones. Brain Res 217:245-263.

Somogyi P, Hodgson AJ, Chubb IW, Penke B, Erdei A (1985) Antisera to $\gamma$-aminobutyric acid. II. Immunocytochemical application to the central nervous system. J Histochem Cytochem 33:240-248.

Van Hoesen GW, Yeterian EH, Lavizzo Mourey R (1981) Widespread corticostriate projections from temporal cortex of the rhesus monkey. J Comp Neurol 199:205-219.

Veenman CL, Reiner A, Hönig MG (1992) Biotinylated dextran amine as an anterograde tracer for single-labeling and double-labeling studies. J Neurosci Methods 41:239-254.

von Krosigk M, Smith AD (1991) Descending projections from the substantia nigra and retrorubral field to the medullary and pontomedullary reticular formation. Eur J Neurosci 3:260-273.

Wurtz RH, Hikosaka O (1986) Role of the basal ganglia in the initiation of saccadic eye movements. Prog Brain Res 64:175-190.

Yelnik J, Percheron G, Francois C (1984) A Golgi analysis of the primate globus pallidus. II. Quantitative morphology and spatial orientation of dendritic arborizations. J Comp Neurol 227:200-213.

Yung KKL, Smith AD, Levey AI, Bolam JP (1996) Synaptic connections between spiny neurons in the neostriatum of the rat: evidence from dopamine receptor and neuropeptide immunostaining. Eur J Neurosci 8:861-869. 